(R P 365)

\title{
A CORRELATED COLOR TEMPERATURE FOR ILLUMINANTS
}

\author{
By Raymond Davis
}

\section{ABSTRACT}

As has long been known, most of the artificial and natural illuminants do not match exactly any one of the Planckian colors. Therefore, strictly speaking, they can not be assigned a color temperature. A color of this type may, however, be correlated with a representative Planckian color.

The method of determining correlated color temperature described in this paper consists in comparing the relative luminosities of each of the three primary red, green, and blue components of the source with similar values for the Planckian series.

With such a comparison three component temperatures are obtained; that is, the red component of the source corresponds with that of the Planckian radiator at one temperature, its green component with that of the Planckian radiator at a second temperature, and its blue component with that of the Planckian radiator at a third temperature. The average of these three component temperatures is designated as the correlated color temperature of the source. The mean deviation of the component temperatures from the average temperature is used as a basis for specifying the color (chromaticity) departure of the source from that of the Planckian radiator at the correlated color temperature. The conjunctive wave length indicates the kind of color departure.

\section{CONTENTS}

I. Introduction

II. The proposed method

III. Procedure

1. The Planckian radiator evaluated in terms of relative juminosity of the primary components

2. Computation of the correlated color temperature

3. Calculation of color departure in terms of sensation steps...- 672

4. Determination of the conjunctive wave length ........ 674

IV. Characteristics of correlated color temperatures.... 674

1. Color departure and the isotemperature line.......... 674

2. Conjunctive wave length as a function of correlated color temperature... 677

V. Correlated color temperature data for Davis-Gibson filters $2,450^{\circ}$ to $3,500^{\circ} \mathrm{K}$. and $2,450^{\circ}$ to $6,500^{\circ} \mathrm{K}$ VI. Bibliography

\section{INTRODUCTION}

The method of describing the color of an illuminant in terms of the temperature at which a black body will have the same color was apparently first used by Hyde $(1)^{1}$ in 1911 . Since that time, this method of assigning "color temperatures" to incandescent bodies has

1 Numbers in parentheses, followed occasionally by page references, refer to the bibliography at the end of the paper. 
come into general use. It furnishes a much simpler description of the chromaticity than a table giving spectral energy values. A definition of color temperature which is in accord with the common usage of the term is that of Priest (2) which follows. The color temperature of a light source is "the temperature at which a Planckian radiator" would emit radiant energy competent to evoke a color of the same quality as that evoked by the radiant energy from the source in question." This definition is seldom strictly applicable because probably none of the common illuminants evokes a color matching exactly any of those evoked by the Planckian radiator; therefore, under this definition artificial and natural illuminants can not strictly be assigned color temperatures. In such cases the specification aims to fix upon that Planckian temperature which furnishes the minimum difference in color; that is, that color temperature is specified which most nearly matches the given source. For a satisfactory specification the color difference involved must then be evaluated in readily appreciable terms.

In this paper are considered particularly illuminants which do not match exactly any of the Planckian colors, but whose color may be definitely correlated with a Planckian color. The above definition of color temperature may then be extended as follows: The "ideal correlated color temperature" of a light source is the absolute temperature at which the Planckian radiator emits radiant energy competent to evoke a color which, of all Planckian colors, most closely approximates the color evoked by the source in question. Strictly speaking, ideal correlated color temperatures can not be computed because at the present time no computational method can be successfully defended on theoretical grounds. Ideal correlated color temperatures may be approximated by direct observation under certain specific conditions; that is, the values may differ from the ideal correlated color temperature by the observational uncertainty. Accordingly, we shall call all color-temperature values representing non-Planckian colors "correlated color temperatures" regardless of the method by which they were obtained provided they are intended to approximate the ideal correlated color temperature.

The correlated color temperature derived by the method described in this paper is believed to give a Planckian temperature which is at least very close to the nearest match with the given source. The description is then completed by giving the kind and magnitude of the color difference. The correlated color temperature by this method approaches the true color temperature as a limit, as an exact color match between the Planckian radiator and the given source is approached; hence, in this respect there can be no question as to the applicability of the method. The degree of color departure ${ }^{3}$ of the given source from that of the Planckian radiator at the correlated temperature is then expressible definitely in terms of trilinear coor-

\footnotetext{
$2 \mathrm{By}$ defining color temperature in terms of a Planckian radiator, Planck's distribution formula, as given in B. S. Misc. Pub. No. 56, is used as an energy basis irrespective of the question as to whether or not it perfectly describes black body radiation. See also the footnote 3 .

. In accord with the definition of "chromaticity" given in the Report of Committee on Colorimetry for $1920-21$, ((5) p. 535) "chromaticity" departure should be used instead of color departure since brightness is not to be included in this concept. "But by the same token "chromaticity temperature" should then be used instead of "color temperature." The same reservation, of course, applies to the definition of color temperature and of the ideal correlated color temperature. Throughout this paper it will be understood that when the terms color departure, color match, color difference, etc., are used, chromaticity departure, chrowaticity match, chromaticity difference, etc., are meant.
} 
dinates; or, proportionately in terms of the Planckian termperature scale in the immediate vicinity; or, by the number of sensation steps (least perceptible differences) involved. The kind of color departure of the source in question from that of the Planckian radiator at the correlated temperature is given in terms of the wave length of homogeneous light which added to or subtracted from the given source will furnish a perfect match with the Planckian radiator at the correlated temperature.

To evaluate the colors of illuminants in terms of color temperature it is necessary to adopt as a reference basis, a set of spectral energy distributions as a function of the temperature of the radiator. For this purpose it is customary to use the energy distributions calculated by Planck's well-known formula. For each of these energy distributions a single variable is usually derived which serves to represent the Planckian distribution. The corresponding quantity is then computed for the energy distribution of the illuminant. By comparing this value with the series of Planckian values the color temperature of the given distribution may be estimated. Single variables which have been used are $(a)$ the slope of the relative spectral energy curve in the visual part of the spectrum, $(b)$ the wave-length coordinate of the maximum of the energy curve (Wien's displacement law), and $(c)$ the wave-length coordinate of the center of gravity of the luminosity curve of the source ( $\lambda_{c}$ method) (2). Forsythe (3) has used the red-blue ratio, which in effect is equivalent to slope; and the method involving Wien's law is commonly used for assigning stellar temperatures. The first two of these methods are suitable only for grading energy distributions giving smooth curves similar to those of the Planckian radiator. They are not at all suitable for grading irregular or non-Planckian energy distributions in terms of color temperatures. The $\lambda_{c}$ method employed by Priest has been used at the National Bureau of Standards and by others for some time. The proposed method appears satisfactory from the standpoint of accuracy and recommends itself through its relative completeness.

Among the light filters designed by Davis and Gibson (4) are two which were intended to duplicate more or less accurately a series of Planckian colors by varying the color temperature of the light source from $2,000^{\circ}$ to $3,100^{\circ} \mathrm{K}$. With one of the filters the color temperature range is from $2,650^{\circ}$ to $5,000^{\circ} \mathrm{K}$. and with the other, from $4,000^{\circ}$ to $18,600^{\circ} \mathrm{K}$. The locus of colors evolied by the lamp-and-filter combination crosses the Planckian locus of colors at a small angle, the point of intersection being in one case at $3,500^{\circ} \mathrm{K}$., and in the other at $6,500^{\circ} \mathrm{K}$. In both of these instances the light source, is, by design, at $2,450^{\circ} \mathrm{K}$. Thus, at one point in each case the filter combined with the source at $2,450^{\circ} \mathrm{K}$. evokes a color which coincides with a Planckian color, and for all other color temperatures of the light source the agreement is more or less imperfect, the imperfection increasing with the departure of the light source from $2,450^{\circ} \mathrm{K}$. If these two filters are to serve the purpose for which they were designed, not only the correlated color temperatures obtainable therewith at the various light-source temperatures should be specified, but also the degree of the approximation. This was the immediate spur to the present undertaking. 


\section{THE PROPOSED METHOD}

The method of correlating a non-Planckian source with a Planckian source followed here, may be described by plotting (trilinear coordinates) the locus of Planckian colors, and inscribing on this curve the corresponding temperature scale. The color of the Planckian radiator at any given temperature is then specified equally definitely by the trilinear coordinates or by the temperature.

Colors off the Planckian locus are correlated with this temperature scale by averaging the three temperatures (primary component tem-

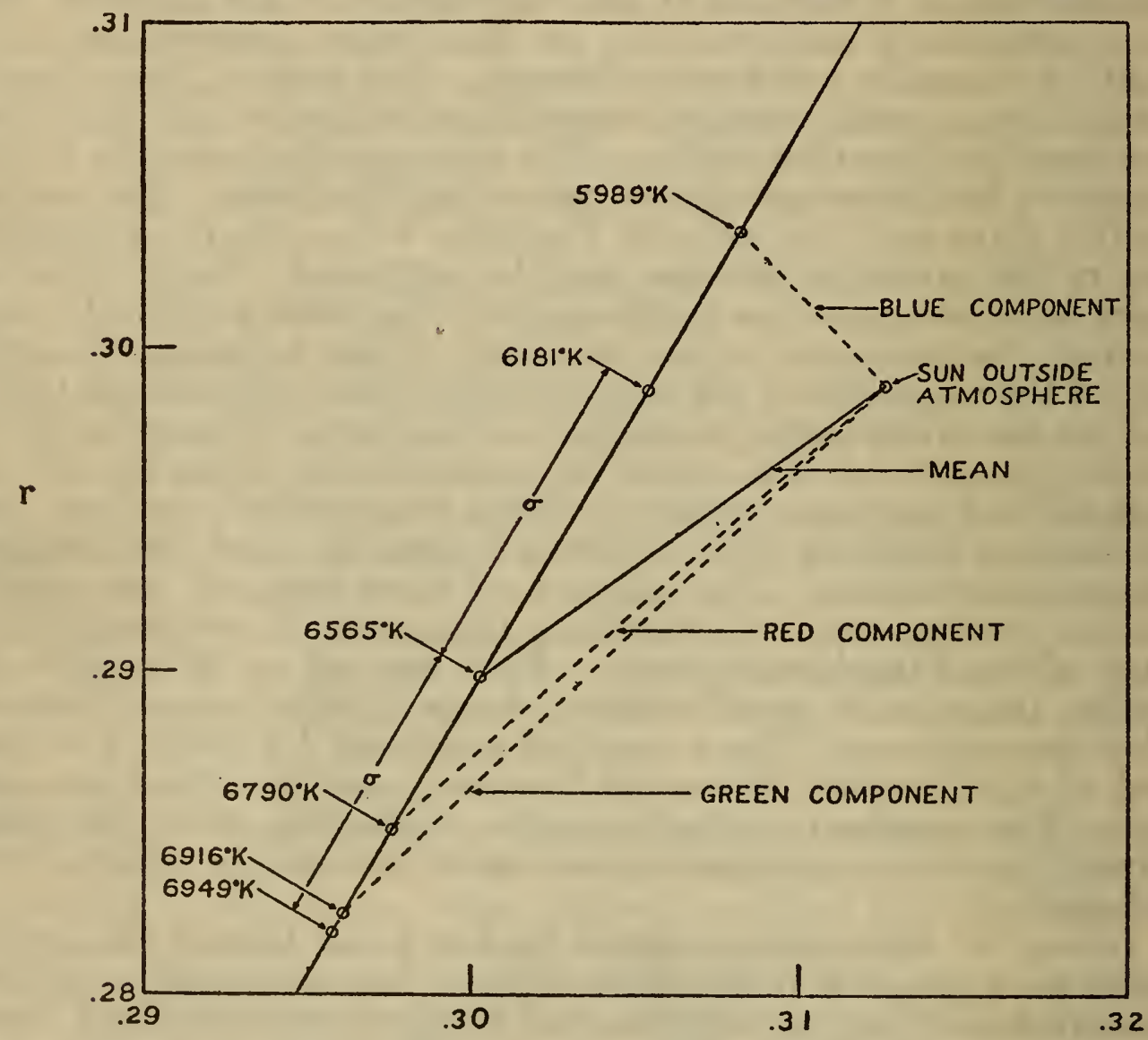

9

FIGURE 1.-A section of the trilinear diagram ( $r-g$ projection) showing the colorimetric positions of the component temperatures, the mean temperature, and the mean deviation $(\sigma)$ for sunlight outside the earth's atmosphere computed from Abbot's 1917 data (4)

peratures) at which the Planckian color has, respectively, in terms of luminosity the same fractional part of primary "red," "green," and "blue" as the non-Planckian color. This average temperature (correlated color temperature) is used to associate non-Planckian colors with some definite color temperature. Points representing colors having the same correlated color temperature form lines on the trilinear diagram (isotemperature lines) intersecting the Planckian locus at the point where the temperature of the Planckian radiator and the correlated color temperature of the source are identical. The system of isotemperature lines fills the color area with nonintersecting lines which, sufficiently near the Planckian locus, may be treated as parallel 
straight lines. These are more widely spaced at lower temperatures $\left(2,000^{\circ} \mathrm{K}\right.$.) crowding closely at the higher temperatures $\left(20,000^{\circ} \mathrm{K}\right.$.). See for example Figure 3.

Figure 1 is a restricted plot which shows the correlation of the color of the sun outside the earth's atmosphere with that of the Planckian radiator at $6,565^{\circ} \mathrm{K}$. As indicated, the sun's red component corresponds in luminosity to that of a Planckian radiator at $6,790^{\circ}$, its green component to that of a Planckian radiator at $6,916^{\circ}$, and its blue component to that of a Planckian radiator at $5,989^{\circ} \mathrm{K}$. By the

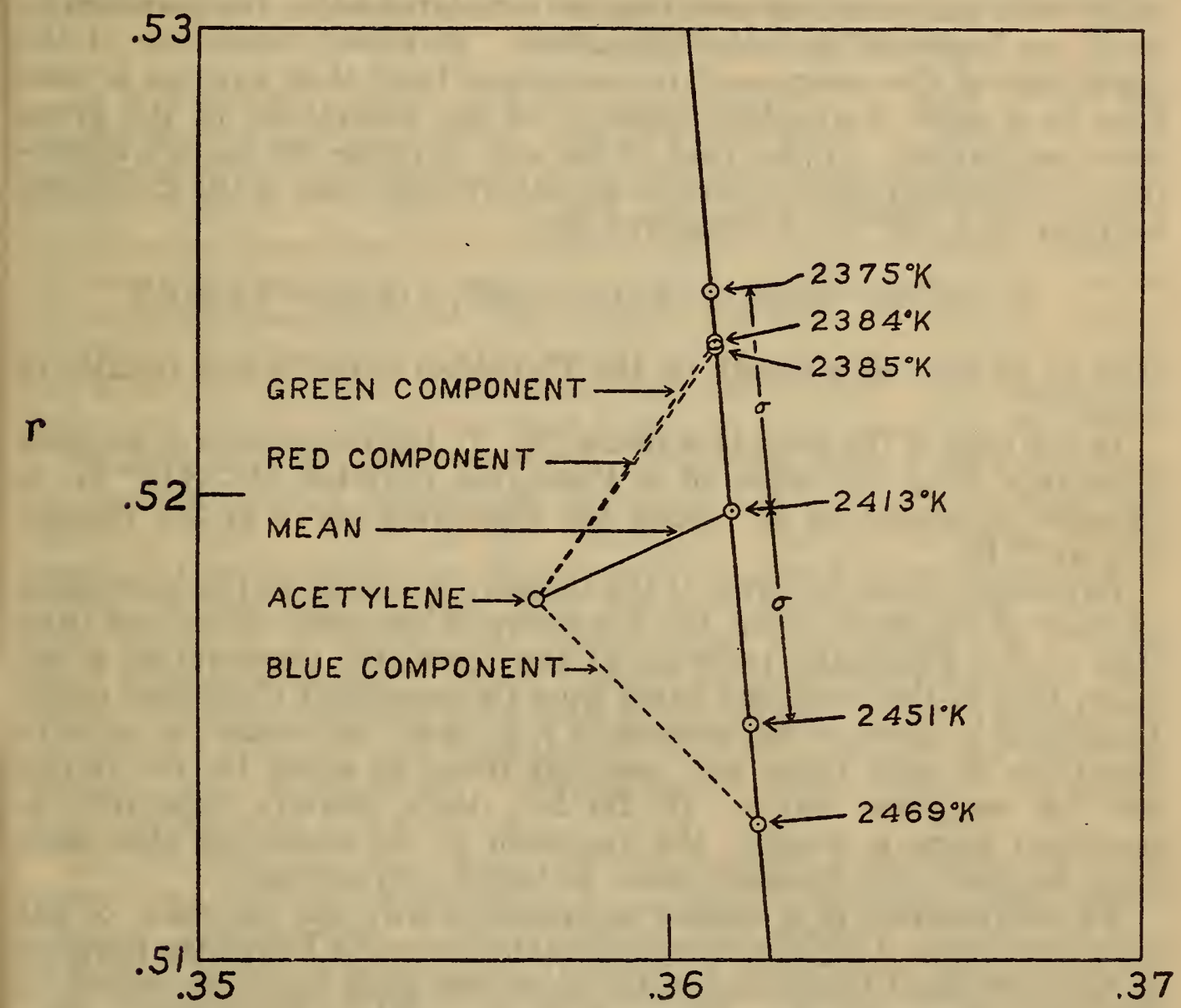

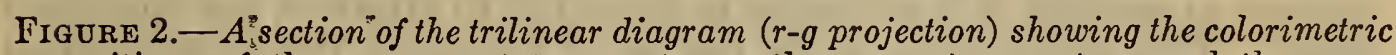
positions of the component temperatures, the mean temperature and the mean deviation $(\sigma)$ for the energy distribution of the acetylene flame computed from Coblentz's data

present method the mean of these three temperatures, $6,565^{\circ} \mathrm{K}$., is the correlated color temperature of the sun. The line which joins the sun's colorimetric position with that of the Planckian radiator at $6,565^{\circ}$ is an isotemperature line along which the color changes by magnitudes approximately proportional to distance. Likewise, Figure 2 illustrates the correlated color temperature $\left(2,413^{\circ} \mathrm{K}\right.$.) of the acetylene flame and its color departure from the correlated Planckian color.

If these magnitudes of color departure in the specific direction and for the specific locations be evaluated once in terms of sensation units, 
they may be used as a basis for evaluating like directed color changes in the immediate neighborhood. There is, however, no a priori reason for applying the evaluation of sensation magnitudes in one direction to those in another direction, in fact, they are known to be different. The same reservation applies to points in different neighborhoods even though the direction of the departure be the same.

We may now consider expressing the degree of color departure of a given source from that of the Planckian radiator at the correlated temperature. The length of the line connecting the non-Planckian point with the point representing its correlated color temperature is, itself, an index of the color departure. However, the mean of the deviations of the component temperatures from their average is used here as a more convenient measure of the magnitude of the given color separation. In the case of the sun (outside the earth's atmosphere) the magnitude of its color departure from that of the Planckian radiator at $6,565^{\circ} \mathrm{K}$. is measured by

$$
\sigma=[(6,790-6,565)+(6,916-6,565)+(6,565-5,989)] / 3
$$

that is, by $384^{\circ}$ as a length on the Planckian curve in the vicinity of $6,565^{\circ} \mathrm{K}$.

In the case of the acetylene flame (fig. 2), the magnitude of its color departure from the color of a Planckian radiator at $2,413^{\circ} \mathrm{K}$. is likewise measured by $38^{\circ}$ along the Planckian curve in the vicinity of $2,413^{\circ} \mathrm{K}$.

Expressed, then, in terms of the temperature scale in the immediate vicinity of the given color, the departure of the color of the sun from that of the Planckian radiator at the correlated temperature is ten times that of the acetylene flame from its correlated Planckian point. Expressed in terms of differences in $r, g$, and $b$ primaries the relative departure is only three and one-half times as much for the sun as for the acetylene flame. If, further, their relative departure in sensation steps is sought, the variation of the sensation scale with both location and direction must be taken into account.

To characterize in a readily appreciable way the direction of the color departure of a given source from the color of a Planckian radiator at the correlated temperature, the spectrum locus also is inscribed on the color diagram, as in Figure 3, for example. From that region near the black-body curve where the isotemperature line may be considered as straight it is extended in direction to intersect the spectrum locus. This intersection gives the wave length of the homogeneous light (conjunctive wave length) which must be added to, or subtracted from, as the case may be, the given source in order to make it match exactly in chromaticity the Planckian radiator at the correlated temperature. In the case of the sun, this conjunctive wave length is about $565 \mathrm{~m} \mu$; for the acetylene flame it is slightly more than 580 $\mathrm{m} \mu$. For a color temperature of $1,800^{\circ}$, it is $586 \mathrm{~m} \mu$; and for $15,000^{\circ}$, it is near $560 \mathrm{~m} \mu$. These limits correspond to only a moderate deviation of the conjunctive wave-length lines from parallelism.

Color points beyond the region in which the isotemperature lines are practically straight are specified in exactly the same way as those falling within this region. The conjunctive wave length serves just as definitely in these cases to specify the direction of the color departure of the source in question from the color of the Planckian radiator 
at the correlated color temperature; but this direction is slightly different from that for nearer color points, hence could not be expected to coincide with the direction of least color difference in terms of sensation change if it does for nearer points. However, the range of colors expressible in terms of correlated color temperature should obviously be restricted to near-Planckian colors

\section{PROCEDURE}

\section{THE PLANCKIAN RADIATOR EVALUATED IN TERMS OF RELATIVE LUMINOSITY OF THE PRIMARY COMPONENTS}

As a necessary basis, trilinear coordinates, $r, g$, and $b$, for the Planckian radiator at various temperatures were computed by the method described in the report of the committee on colorimetry of the Optical Society of America (5) and elsewhere (Abney, Ives, Guild). In the paper describing Davis-Gibson filters (4) colorimetric computations on this basis, with mean noon sunlight represented at the center of the color triangle, have been carried out not only for the energy distributions obtained with the various lamp and filter combinations, but also for Planckian distributions between $1,600^{\circ}$ and $20,000^{\circ} \mathrm{K}$. Reference should be made to that paper for the basic data used here.

It is the generally accepted view that the products of the excitations by their respective luminosity coefficients should sum to yield the visibility curve. Because the luminosity coefficients given in the report of the committee on colorimetry (5) were not considered satisfactory in this respect, new ones were computed, by least squares, from the O. S. A. excitations, extrapolated (6), adjusted to "mean sun" and the standard Gibson-Tyndall recommended visibility data (7) in a manner differing from that used by Judd (8) only in that the equations contained the requirement that the sum of the three coefficients shall equal unity. The values so obtained are $L_{r}=0.45014$, $L_{o}=0.54417$, and $L_{b}=0.00569$.

The products of $r, g$, and $\dot{b}$ for any energy distribution by their respective luminosity coefficients $L_{r}, L_{\theta}$, and $L_{b}$ give the three relative primary luminosities. Computing in this manner values for the Planckian series, it is seen that the sum of these three varies with the color temperature; which is to be expected. To give them on a basis of equal luminosities $r L_{r}, g L_{o}$, and $b L_{b}$ are, for each temperature, multiplied by a factor $\omega$ such that the sum is one-third.

In Table 1 are given then $\omega r L_{r}, \omega g L_{g}$, and $\omega b L_{b}$ for Planckian energy distributions obtained by computation and by graphical interpolation from their respective $r, g$, and $b$ values. 
TABLE 1.-Table to facilitate the computation of correlated color temperatures

The $r, g$, and $b$ values of the energy distribution (computed as illustrated in B. S. Mis. Pub. No. 114, Figure 12) are multiplied by the respectivo luminosity coefficients $\left(L_{r}=0.45014, L_{g}=0.54417, L_{b}=0.00569\right)$. The resulting values are adjusted by a factor $(\omega)$ so that their sum is 0.33333 .

\begin{tabular}{|c|c|c|c|c|c|c|c|}
\hline $\begin{array}{l}\text { Color } \\
\text { temper- } \\
\text { ature }\end{array}$ & $\omega r L_{r}$ & $\omega g L_{g}$ & $\omega b L_{b}$ & $\begin{array}{l}\text { Color } \\
\text { temper- } \\
\text { ature }\end{array}$ & $\omega r L$ & $\omega g L_{\theta}$ & $\omega b L_{b}$ \\
\hline $\begin{array}{r}\circ K \\
1,600 \\
10 \\
20 \\
30 \\
40\end{array}$ & $\begin{array}{r}0.20788 \\
.20741 \\
.20593 \\
.20546 \\
.20599\end{array}$ & $\begin{array}{r}0.12530 \\
.12577 \\
.12624 \\
.12672 \\
.12718\end{array}$ & $\begin{array}{r}0.000152 \\
.000156 \\
.000159 \\
.000162 \\
.000166\end{array}$ & $\begin{array}{r}\circ K \\
2,500 \\
20 \\
40 \\
60 \\
80\end{array}$ & $\begin{array}{r}0.17895 \\
.17854 \\
.17813 \\
.17773 \\
.17733\end{array}$ & $\begin{array}{r}0.15382 \\
.15421 \\
.15461 \\
.15500 \\
.15539\end{array}$ & $\begin{array}{r}0.000571 \\
.000582 \\
.000593 \\
.000604 \\
.000616\end{array}$ \\
\hline $\begin{array}{r}1,650 \\
60 \\
70 \\
80 \\
90\end{array}$ & $\begin{array}{l}.20552 \\
.20505 \\
.20459 \\
.20413 \\
.20369\end{array}$ & $\begin{array}{l}.12765 \\
.12812 \\
.12857 \\
.12902 \\
.12946\end{array}$ & $\begin{array}{l}.000169 \\
.000173 \\
.000176 \\
.000180 \\
.000183\end{array}$ & $\begin{array}{r}2,600 \\
20 \\
40 \\
60 \\
80\end{array}$ & $\begin{array}{l}.17693 \\
.17654 \\
.17615 \\
.17578 \\
.17541\end{array}$ & $\begin{array}{l}.15577 \\
.15614 \\
.15652 \\
.15688 \\
.15725\end{array}$ & $\begin{array}{l}.000627 \\
.000639 \\
.000650 \\
.000661 \\
.000673\end{array}$ \\
\hline $\begin{array}{r}1,700 \\
10 \\
20 \\
30 \\
40\end{array}$ & $\begin{array}{l}.20324 \\
.20281 \\
.20237 \\
.20194 \\
.20152\end{array}$ & $\begin{array}{l}.12990 \\
.13034 \\
.13077 \\
.13120 \\
.13161\end{array}$ & $\begin{array}{l}.000187 \\
.000191 \\
.000194 \\
.000198 \\
.000202\end{array}$ & $\begin{array}{r}2,700 \\
20 \\
40 \\
60 \\
80\end{array}$ & $\begin{array}{r}.17505 \\
.17470 \\
.17435 \\
.17401 \\
.17368\end{array}$ & $\begin{array}{l}.15760 \\
.15794 \\
.15828 \\
.15861 \\
.15893\end{array}$ & $\begin{array}{l}.000684 \\
.000696 \\
.000707 \\
.000719 \\
.000730\end{array}$ \\
\hline $\begin{array}{r}1,750 \\
60 \\
70 \\
80 \\
90\end{array}$ & $\begin{array}{l}.20110 \\
.20059 \\
.20029 \\
.19988 \\
.19948\end{array}$ & $\begin{array}{l}.13203 \\
.13244 \\
.13284 \\
.13324 \\
.13363\end{array}$ & $\begin{array}{l}.000205 \\
.000210 \\
.000214 \\
.000218 \\
.000222\end{array}$ & $\begin{array}{r}2,800 \\
20 \\
40 \\
60 \\
80\end{array}$ & $\begin{array}{r}.17334 \\
.17301 \\
.17268 \\
.17236 \\
.17203\end{array}$ & $\begin{array}{l}.15925 \\
.15957 \\
.15989 \\
.16020 \\
.16051\end{array}$ & $\begin{array}{l}.000742 \\
.000753 \\
.000765 \\
.000777 \\
.000788\end{array}$ \\
\hline $\begin{array}{r}1,800 \\
10 \\
20 \\
30 \\
40\end{array}$ & $\begin{array}{l}.19909 \\
.19870 \\
.19831 \\
.19793 \\
.19755\end{array}$ & $\begin{array}{l}.13402 \\
.13440 \\
.13478 \\
.13516 \\
.13554\end{array}$ & $\begin{array}{l}.000226 \\
.000230 \\
.000234 \\
.000238 \\
.000243\end{array}$ & $\begin{array}{r}2,900 \\
20 \\
40 \\
60 \\
80\end{array}$ & $\begin{array}{l}.17172 \\
.17142 \\
.17112 \\
.17082 \\
.17053\end{array}$ & $\begin{array}{l}.16081 \\
.16111 \\
.16140 \\
.16168 \\
.16196\end{array}$ & $\begin{array}{l}.000800 \\
.000812 \\
.000824 \\
.000835 \\
.000847\end{array}$ \\
\hline $\begin{array}{r}1,850 \\
60 \\
70 \\
80 \\
90\end{array}$ & $\begin{array}{l}.19716 \\
.19679 \\
.19643 \\
.19606 \\
.19570\end{array}$ & $\begin{array}{l}.13591 \\
.13628 \\
.13664 \\
.13700 \\
.13736\end{array}$ & $\begin{array}{l}.000247 \\
.000251 \\
.000255 \\
.000259 \\
.000264\end{array}$ & $\begin{array}{r}3,000 \\
20 \\
40 \\
60 \\
80\end{array}$ & $\begin{array}{l}.17023 \\
.16994 \\
.16965 \\
.16937 \\
.16908\end{array}$ & $\begin{array}{l}.16225 \\
.16252 \\
.16280 \\
.16307 \\
.16334\end{array}$ & $\begin{array}{l}.000859 \\
.000871 \\
.000883 \\
.000895 \\
.000907\end{array}$ \\
\hline $\begin{array}{r}1,900 \\
10 \\
20 \\
30 \\
40\end{array}$ & $\begin{array}{l}.19535 \\
.19499 \\
.19464 \\
.19429 \\
.19395\end{array}$ & $\begin{array}{l}.13772 \\
.13807 \\
.13842 \\
.13877 \\
.13910\end{array}$ & $\begin{array}{l}.000258 \\
.000272 \\
.000277 \\
.000281 \\
.000286\end{array}$ & $\begin{array}{r}3,100 \\
20 \\
40 \\
60 \\
80\end{array}$ & $\begin{array}{l}.16880 \\
.16853 \\
.16827 \\
.16800 \\
.16773\end{array}$ & $\begin{array}{l}.16361 \\
.16387 \\
.16413 \\
.16438 \\
.16464\end{array}$ & $\begin{array}{l}.000919 \\
.000930 \\
.000942 \\
.000954 \\
.000966\end{array}$ \\
\hline $\begin{array}{r}1,950 \\
60 \\
70 \\
80 \\
90\end{array}$ & $\begin{array}{l}.19361 \\
.19327 \\
.19293 \\
.19260 \\
.19227\end{array}$ & $\begin{array}{l}.13944 \\
.13977 \\
.14010 \\
.14043 \\
.14075\end{array}$ & $\begin{array}{l}.000290 \\
.000295 \\
.000299 \\
.000304 \\
.000308\end{array}$ & $\begin{array}{r}3,200 \\
20 \\
40 \\
60 \\
80\end{array}$ & $\begin{array}{l}.16747 \\
.16722 \\
.16697 \\
.16672 \\
.16648\end{array}$ & $\begin{array}{r}.16488 \\
.16512 \\
.16537 \\
.16560 \\
.16583\end{array}$ & $\begin{array}{l}.000977 \\
.000989 \\
.001001 \\
.001013 \\
.001024\end{array}$ \\
\hline $\begin{array}{r}2,000 \\
20 \\
40 \\
60 \\
80\end{array}$ & $\begin{array}{l}.19195 \\
.19129 \\
.19056 \\
.19004 \\
.18944\end{array}$ & $\begin{array}{l}.14107 \\
.14171 \\
.14233 \\
.14294 \\
.14354\end{array}$ & $\begin{array}{l}.000313 \\
.000322 \\
.000332 \\
.000342 \\
.000351\end{array}$ & $\begin{array}{r}3,300 \\
20 \\
40 \\
60 \\
80\end{array}$ & $\begin{array}{l}.16623 \\
.16599 \\
.16576 \\
.16553 \\
.16529\end{array}$ & $\begin{array}{l}.16607 \\
.16629 \\
.16652 \\
.16674 \\
.16696\end{array}$ & $\begin{array}{l}.001036 \\
.001048 \\
.001059 \\
.001071 \\
.001082\end{array}$ \\
\hline $\begin{array}{r}2,100 \\
20 \\
40 \\
60 \\
80\end{array}$ & $\begin{array}{l}.18885 \\
.18827 \\
.18770 \\
.18714 \\
.18659\end{array}$ & $\begin{array}{l}.14412 \\
.14470 \\
.14525 \\
.14579 \\
.14634\end{array}$ & $\begin{array}{l}.000361 \\
.000371 \\
.000381 \\
.000391 \\
.000401\end{array}$ & $\begin{array}{r}3,400 \\
20 \\
40 \\
60 \\
80\end{array}$ & $\begin{array}{l}.16507 \\
.16484 \\
.16462 \\
.16440 \\
.16419\end{array}$ & $\begin{array}{l}.16718 \\
.16739 \\
.16759 \\
.16780 \\
.16800\end{array}$ & $\begin{array}{l}.001094 \\
.001106 \\
.001117 \\
.001129 \\
.001141\end{array}$ \\
\hline $\begin{array}{r}2,200 \\
20 \\
40 \\
60 \\
80\end{array}$ & $\begin{array}{l}.18606 \\
.18553 \\
.18499 \\
.18447 \\
.18397\end{array}$ & $\begin{array}{l}.14686 \\
.14735 \\
.14790 \\
.14841 \\
.14891\end{array}$ & $\begin{array}{l}.000411 \\
.000421 \\
.000432 \\
.000442 \\
.000452\end{array}$ & $\begin{array}{r}3,500 \\
20 \\
40 \\
60 \\
80\end{array}$ & $\begin{array}{l}.16398 \\
.16377 \\
.16355 \\
.16335 \\
.16315\end{array}$ & $\begin{array}{l}.16820 \\
.16841 \\
.16860 \\
.16879 \\
.16898\end{array}$ & $\begin{array}{l}.001152 \\
.001164 \\
.001176 \\
.001187 \\
.001199\end{array}$ \\
\hline $\begin{array}{r}2,300 \\
20 \\
40 \\
60 \\
80\end{array}$ & $\begin{array}{l}.18347 \\
.18298 \\
.18252 \\
.18204 \\
.18157\end{array}$ & $\begin{array}{l}.14939 \\
.14987 \\
.15033 \\
.15080 \\
.15124\end{array}$ & $\begin{array}{l}.000463 \\
.000473 \\
.000484 \\
.000494 \\
.000505\end{array}$ & $\begin{array}{r}3,600 \\
20 \\
40 \\
60 \\
80\end{array}$ & $\begin{array}{l}.16295 \\
.16274 \\
.16255 \\
.16236 \\
.16217\end{array}$ & $\begin{array}{l}.16917 \\
.16937 \\
.16955 \\
.16973 \\
.16991\end{array}$ & $\begin{array}{l}.001210 \\
.001222 \\
.001233 \\
.001245 \\
.001256\end{array}$ \\
\hline $\begin{array}{r}2,400 \\
20 \\
40 \\
60 \\
80\end{array}$ & $\begin{array}{l}.18112 \\
.18068 \\
.18024 \\
.17980 \\
.17937\end{array}$ & $\begin{array}{l}.15169 \\
.15212 \\
.15255 \\
.15298 \\
.15340\end{array}$ & $\begin{array}{l}.000516 \\
.000527 \\
.000538 \\
.000549 \\
.000560\end{array}$ & $\begin{array}{r}3,700 \\
20 \\
40 \\
60 \\
80\end{array}$ & $\begin{array}{l}.16197 \\
.16178 \\
.16160 \\
.16141 \\
.16123\end{array}$ & $\begin{array}{l}.17009 \\
.17027 \\
.17044 \\
.17062 \\
.17079\end{array}$ & $\begin{array}{l}.001268 \\
.001280 \\
.001291 \\
.001303 \\
.001314\end{array}$ \\
\hline
\end{tabular}


TABLE 1.-Table to facilitate the computation of correlated color temperatures-Con.

\begin{tabular}{|c|c|c|c|c|c|c|c|}
\hline $\begin{array}{l}\text { Color } \\
\text { temper- } \\
\text { ature }\end{array}$ & $\omega r L_{r}$ & $\omega g L_{0}$ & $\omega b L_{b}$ & $\begin{array}{l}\text { Color } \\
\text { temper- } \\
\text { ature }\end{array}$ & $\omega r L_{r}$ & $\omega g L$ & $\omega b L_{b}$ \\
\hline \begin{tabular}{rr|}
$\circ K$ \\
3,800 \\
20 \\
40 \\
60 \\
80
\end{tabular} & $\begin{array}{r}0.16104 \\
.16086 \\
.16068 \\
.16050 \\
.16033\end{array}$ & $\begin{array}{r}0.17096 \\
.17113 \\
.17130 \\
.17147 \\
.17163\end{array}$ & $\begin{array}{r}0.001326 \\
.001337 \\
.001348 \\
.001360 \\
.001371\end{array}$ & $\begin{array}{r}\circ K \\
5,100 \\
20 \\
40 \\
60 \\
80\end{array}$ & $\begin{array}{r}0.15232 \\
.15222 \\
.15213 \\
.15203 \\
.15193\end{array}$ & $\begin{array}{r}0.17900 \\
.17908 \\
.17917 \\
.17926 \\
.17935\end{array}$ & $\begin{array}{r}0.002007 \\
.002017 \\
.002027 \\
.002036 \\
.002046\end{array}$ \\
\hline $\begin{array}{r}3,900 \\
20 \\
40 \\
60 \\
80\end{array}$ & $\begin{array}{l}.16016 \\
.15998 \\
.15981 \\
.15964 \\
.15947\end{array}$ & $\begin{array}{l}.17179 \\
.17196 \\
.17212 \\
.17227 \\
.17243\end{array}$ & $\begin{array}{l}.001382 \\
.001394 \\
.001405 \\
.001416 \\
.001427\end{array}$ & $\begin{array}{r}5,200 \\
20 \\
40 \\
60 \\
80\end{array}$ & $\begin{array}{l}.15184 \\
.15174 \\
.15165 \\
.15156 \\
.15147\end{array}$ & $\begin{array}{l}.17943 \\
.17952 \\
.17960 \\
.17968 \\
.17977\end{array}$ & $\begin{array}{l}.002055 \\
.002064 \\
.002073 \\
.002083 \\
.002093\end{array}$ \\
\hline $\begin{array}{r}4,000 \\
20 \\
40 \\
60 \\
80\end{array}$ & $\begin{array}{l}.15931 \\
.15914 \\
.15898 \\
.15882 \\
.15866\end{array}$ & $\begin{array}{r}.17258 \\
.17274 \\
.17288 \\
.17304 \\
.17318\end{array}$ & $\begin{array}{l}.001438 \\
.001450 \\
.001461 \\
.001472 \\
.001483\end{array}$ & $\begin{array}{r}5,300 \\
20 \\
40 \\
60 \\
80\end{array}$ & $\begin{array}{l}.15138 \\
.15128 \\
.15119 \\
.15111 \\
.15102\end{array}$ & $\begin{array}{l}.17986 \\
.17994 \\
.18002 \\
.18010 \\
.18018\end{array}$ & $\begin{array}{l}.002102 \\
.002111 \\
.002120 \\
.002129 \\
.002138\end{array}$ \\
\hline $\begin{array}{r}4,100 \\
20 \\
40 \\
60 \\
80\end{array}$ & $\begin{array}{l}.15850 \\
.15834 \\
.15818 \\
.15803 \\
.15788\end{array}$ & $\begin{array}{l}.17334 \\
.17349 \\
.17363 \\
.17377 \\
.17391\end{array}$ & $\begin{array}{l}.001494 \\
.001505 \\
.001516 \\
.001527 \\
.001538\end{array}$ & $\begin{array}{r}5,400 \\
20 \\
40 \\
60 \\
80\end{array}$ & $\begin{array}{l}.15093 \\
.15084 \\
.15075 \\
.15066 \\
.15057\end{array}$ & $\begin{array}{l}.18026 \\
.18034 \\
.18042 \\
.18050 \\
.18058\end{array}$ & $\begin{array}{l}.002148 \\
.002157 \\
.002166 \\
.002175 \\
.002184\end{array}$ \\
\hline $\begin{array}{r}4,200 \\
20 \\
40 \\
60 \\
80\end{array}$ & $\begin{array}{l}.15773 \\
.15758 \\
.15743 \\
.15729 \\
.15715\end{array}$ & $\begin{array}{l}.17405 \\
.17419 \\
.17433 \\
.17446 \\
.17459\end{array}$ & $\begin{array}{l}.001549 \\
.001560 \\
.001571 \\
.001581 \\
.001592\end{array}$ & $\begin{array}{r}5,500 \\
20 \\
40 \\
60 \\
80\end{array}$ & $\begin{array}{l}.15049 \\
.15041 \\
.15032 \\
.15023 \\
.15016\end{array}$ & $\begin{array}{l}.18065 \\
.18073 \\
.18081 \\
.18088 \\
.18095\end{array}$ & $\begin{array}{l}.002193 \\
.002203 \\
.002212 \\
.002221 \\
.002229\end{array}$ \\
\hline $\begin{array}{r}4,300 \\
20 \\
40 \\
60 \\
80\end{array}$ & $\begin{array}{l}.15701 \\
.15687 \\
.15673 \\
.15659 \\
.15646\end{array}$ & $\begin{array}{l}.17472 \\
.17485 \\
.17498 \\
.17511 \\
.17523\end{array}$ & $\begin{array}{l}.001603 \\
.001613 \\
.001624 \\
.001635 \\
.001645\end{array}$ & $\begin{array}{r}5,600 \\
20 \\
40 \\
60 \\
80\end{array}$ & $\begin{array}{l}.15007 \\
.14999 \\
.14991 \\
.14983 \\
.14975\end{array}$ & $\begin{array}{l}.18102 \\
.18110 \\
.18117 \\
.18124 \\
.18132\end{array}$ & $\begin{array}{l}.002237 \\
.002246 \\
.002255 \\
.002264 \\
.002273\end{array}$ \\
\hline $\begin{array}{r}4,400 \\
20 \\
40 \\
60 \\
80\end{array}$ & $\begin{array}{l}.15632 \\
.15618 \\
.15605 \\
.15593 \\
.15579\end{array}$ & $\begin{array}{r}.17536 \\
.17548 \\
.17560 \\
.17572 \\
.17584\end{array}$ & $\begin{array}{l}.001656 \\
.001666 \\
.001677 \\
.001687 \\
.001698\end{array}$ & $\begin{array}{r}5,700 \\
20 \\
40 \\
60 \\
80\end{array}$ & $\begin{array}{l}.14967 \\
.14959 \\
.14951 \\
.14943 \\
.14936\end{array}$ & $\begin{array}{l}.18138 \\
.18145 \\
.18152 \\
.18159 \\
.18166\end{array}$ & $\begin{array}{l}.002282 \\
.002289 \\
.002297 \\
.002306 \\
.002315\end{array}$ \\
\hline $\begin{array}{r}4,500 \\
20 \\
40 \\
60 \\
80\end{array}$ & $\begin{array}{l}.15568 \\
.15554 \\
.15542 \\
.15529 \\
.15517\end{array}$ & $\begin{array}{l}.17595 \\
.17607 \\
.17618 \\
.17630 \\
.17641\end{array}$ & $\begin{array}{l}.001708 \\
.001718 \\
.001729 \\
.001739 \\
.001749\end{array}$ & $\begin{array}{r}5,800 \\
20 \\
40 \\
60 \\
80\end{array}$ & $\begin{array}{l}.14928 \\
.14920 \\
.14913 \\
.14906 \\
.14899\end{array}$ & $\begin{array}{l}.18173 \\
.18179 \\
.18186 \\
.18193 \\
.18199\end{array}$ & $\begin{array}{r}.002324 \\
.002333 \\
.002341 \\
.002349 \\
.002357\end{array}$ \\
\hline $\begin{array}{r}4,600 \\
20 \\
40 \\
60 \\
80\end{array}$ & $\begin{array}{l}.15504 \\
.15493 \\
.15481 \\
.15468 \\
.15456\end{array}$ & $\begin{array}{l}.17653 \\
.17663 \\
.17674 \\
.17686 \\
.17697\end{array}$ & $\begin{array}{l}.001759 \\
.001770 \\
.001780 \\
.001790 \\
.001800\end{array}$ & $\begin{array}{r}5,900 \\
20 \\
40 \\
60 \\
80\end{array}$ & $\begin{array}{l}.14891 \\
.14884 \\
.14877 \\
.14869 \\
.14863\end{array}$ & $\begin{array}{l}.18205 \\
.18211 \\
.18218 \\
.18224 \\
.18230\end{array}$ & $\begin{array}{l}.002366 \\
.002374 \\
.002382 \\
.002390 \\
.002398\end{array}$ \\
\hline $\begin{array}{r}4,700 \\
20 \\
40 \\
60 \\
80\end{array}$ & $\begin{array}{l}.15445 \\
.15433 \\
.15422 \\
.15410 \\
.15398\end{array}$ & $\begin{array}{r}.17707 \\
.17718 \\
.17728 \\
.17739 \\
.17750\end{array}$ & $\begin{array}{l}.001810 \\
.001820 \\
.001830 \\
.001840 \\
.001850\end{array}$ & $\begin{array}{r}6,000 \\
20 \\
40 \\
60 \\
80\end{array}$ & $\begin{array}{l}.14856 \\
.14848 \\
.14841 \\
.14834 \\
.14827\end{array}$ & $\begin{array}{l}.18237 \\
.18243 \\
.18249 \\
.18255 \\
.18261\end{array}$ & $\begin{array}{l}.002407 \\
.002415 \\
.002423 \\
.002430 \\
.002438\end{array}$ \\
\hline $\begin{array}{r}4,800 \\
20 \\
40 \\
60 \\
80\end{array}$ & $\begin{array}{l}.15387 \\
.15377 \\
.15366 \\
.15355 \\
.15344\end{array}$ & $\begin{array}{r}17760 \\
.17770 \\
.17779 \\
.17789 \\
.17799\end{array}$ & $\begin{array}{l}.001860 \\
.001870 \\
.001880 \\
.001890 \\
.001900\end{array}$ & $\begin{array}{r}6,100 \\
20 \\
40 \\
60 \\
80\end{array}$ & $\begin{array}{l}.14821 \\
.14814 \\
.14807 \\
.14801 \\
.14794\end{array}$ & $\begin{array}{r}.18267 \\
.18273 \\
.18279 \\
.18285 \\
.18291\end{array}$ & $\begin{array}{l}.002446 \\
.002454 \\
.002452 \\
.002470 \\
.002478\end{array}$ \\
\hline $\begin{array}{r}4,900 \\
20 \\
40 \\
60 \\
80\end{array}$ & $\begin{array}{l}.15333 \\
.15323 \\
.15312 \\
.15302 \\
.15292\end{array}$ & $\begin{array}{l}.17809 \\
.17818 \\
.17828 \\
.17837 \\
.17846\end{array}$ & $\begin{array}{l}.001910 \\
.001920 \\
.001930 \\
.001940 \\
.001950\end{array}$ & $\begin{array}{r}6,200 \\
20 \\
40 \\
60 \\
80\end{array}$ & $\begin{array}{l}.14788 \\
.14782 \\
.14775 \\
.14768 \\
.14762\end{array}$ & $\begin{array}{l}.18297 \\
.18302 \\
.18309 \\
.18314 \\
.18320\end{array}$ & $\begin{array}{l}.002486 \\
.002494 \\
.002502 \\
.002509 \\
.002517\end{array}$ \\
\hline $\begin{array}{r}5,000 \\
20 \\
40 \\
60 \\
80\end{array}$ & $\begin{array}{l}.15282 \\
.15272 \\
.15262 \\
.15252 \\
.15242\end{array}$ & $\begin{array}{l}.17855 \\
.17864 \\
.17873 \\
.17882 \\
.17891\end{array}$ & $\begin{array}{l}.001960 \\
.001969 \\
.001978 \\
.001988 \\
.001998\end{array}$ & $\begin{array}{r}6,300 \\
20 \\
40 \\
60 \\
80\end{array}$ & $\begin{array}{l}.14755 \\
.14749 \\
.14743 \\
.14737 \\
.14731\end{array}$ & $\begin{array}{l}.18326 \\
.18332 \\
.18337 \\
.18342 \\
.18347\end{array}$ & $\begin{array}{l}.002525 \\
.002533 \\
.002541 \\
.002549 \\
.002556\end{array}$ \\
\hline
\end{tabular}


TABLE 1.-Table to facilitate the computation of correlated color temperatures-Con.

\begin{tabular}{|c|c|c|c|c|c|c|c|}
\hline $\begin{array}{l}\text { Color } \\
\text { temper- } \\
\text { ature }\end{array}$ & $\omega r L_{r}$ & $\omega g L_{0}$ & $\omega b L_{b}$ & $\begin{array}{l}\text { Color } \\
\text { temper- } \\
\text { ature }\end{array}$ & $\omega r L_{p}$ & $\omega g L_{0}$ & $\omega b L_{b}$ \\
\hline $\begin{array}{rc}\circ & K \\
6,400 \\
20 \\
40 \\
60 \\
80\end{array}$ & $\begin{array}{r}0.14724 \\
.14718 \\
.14712 \\
.14705 \\
.14701\end{array}$ & $\begin{array}{r}0.18353 \\
.18358 \\
.18363 \\
.18369 \\
.18374\end{array}$ & $\begin{array}{r}0.002563 \\
.002570 \\
.002577 \\
.002586 \\
.002593\end{array}$ & $\begin{array}{r}\circ K \\
7,700 \\
20 \\
40 \\
60 \\
80\end{array}$ & $\begin{array}{r}0.14398 \\
.14394 \\
.14391 \\
.14387 \\
.14383\end{array}$ & $\begin{array}{r}0.18635 \\
.18638 \\
.18642 \\
.18645 \\
.18648\end{array}$ & $\begin{array}{r}0.003001 \\
.003007 \\
.003013 \\
.003019 \\
.003025\end{array}$ \\
\hline $\begin{array}{r}6,500 \\
20 \\
40 \\
60 \\
80\end{array}$ & $\begin{array}{l}.14695 \\
.14689 \\
.14683 \\
.14677 \\
.14671\end{array}$ & $\begin{array}{l}.18379 \\
.18384 \\
.18390 \\
.18395 \\
.18400\end{array}$ & $\begin{array}{l}.002600 \\
.002607 \\
.002615 \\
.002623 \\
.002630\end{array}$ & $\begin{array}{r}7,800 \\
20 \\
40 \\
60 \\
80\end{array}$ & $\begin{array}{l}.14379 \\
.14375 \\
.14371 \\
.14367 \\
.14363\end{array}$ & $\begin{array}{l}.18652 \\
.18655 \\
.18658 \\
.18662 \\
.18665\end{array}$ & $\begin{array}{l}.003030 \\
.003036 \\
.003042 \\
.003048 \\
.003053\end{array}$ \\
\hline $\begin{array}{r}6,600 \\
20 \\
40 \\
60 \\
80\end{array}$ & $\begin{array}{l}.14666 \\
.14659 \\
.14654 \\
.14648 \\
.14643\end{array}$ & $\begin{array}{l}.18404 \\
.18410 \\
.18415 \\
.18420 \\
.18424\end{array}$ & $\begin{array}{l}.002637 \\
.002644 \\
.002652 \\
.002659 \\
.002666\end{array}$ & $\begin{array}{r}7,900 \\
20 \\
40 \\
60 \\
80\end{array}$ & $\begin{array}{l}.14359 \\
.14356 \\
.14352 \\
.14348 \\
.14345\end{array}$ & $\begin{array}{l}.18668 \\
.18671 \\
.18674 \\
.18678 \\
.18681\end{array}$ & $\begin{array}{l}.003059 \\
.003085 \\
.003070 \\
.003076 \\
.003082\end{array}$ \\
\hline $\begin{array}{r}6,700 \\
20 \\
40 \\
60 \\
80\end{array}$ & $\begin{array}{l}.14637 \\
.14632 \\
.14626 \\
.14621 \\
.14616\end{array}$ & $\begin{array}{l}.18429 \\
.18434 \\
.18439 \\
.18444 \\
.18448\end{array}$ & $\begin{array}{l}.002673 \\
.002680 \\
.002687 \\
.002694 \\
.002701\end{array}$ & $\begin{array}{r}8,000 \\
20 \\
40 \\
60 \\
80\end{array}$ & $\begin{array}{l}.14341 \\
.14337 \\
.14333 \\
.14330 \\
.14326\end{array}$ & $\begin{array}{l}.18684 \\
.18687 \\
.18691 \\
.18694 \\
.18697\end{array}$ & $\begin{array}{l}.003087 \\
.003093 \\
.003098 \\
.003104 \\
.003109\end{array}$ \\
\hline $\begin{array}{r}6,800 \\
20 \\
40 \\
60 \\
80\end{array}$ & $\begin{array}{l}.14610 \\
.14604 \\
.14599 \\
.14594 \\
.14588\end{array}$ & $\begin{array}{l}.18453 \\
.18458 \\
.18462 \\
.18467 \\
.18471\end{array}$ & $\begin{array}{l}.002708 \\
.002715 \\
.002722 \\
.002729 \\
.002736\end{array}$ & $\begin{array}{r}8,100 \\
20 \\
40 \\
60 \\
80\end{array}$ & $\begin{array}{l}.14322 \\
.14319 \\
.14315 \\
.14311 \\
.14308\end{array}$ & $\begin{array}{l}.18700 \\
.18703 \\
.18706 \\
.18709 \\
.18712\end{array}$ & $\begin{array}{l}.003115 \\
.003120 \\
.003126 \\
.003131 \\
.003137\end{array}$ \\
\hline $\begin{array}{r}6,900 \\
20 \\
40 \\
60 \\
80\end{array}$ & $\begin{array}{l}.14583 \\
.14578 \\
.14572 \\
.14568 \\
.14563\end{array}$ & $\begin{array}{l}.18476 \\
.18481 \\
.18485 \\
.18490 \\
.18494\end{array}$ & $\begin{array}{l}.002743 \\
.002750 \\
.002756 \\
.002763 \\
.002770\end{array}$ & $\begin{array}{r}8,200 \\
20 \\
40 \\
60 \\
80\end{array}$ & $\begin{array}{l}.14304 \\
.14300 \\
.14297 \\
.14293 \\
.14290\end{array}$ & $\begin{array}{l}.18715 \\
.18718 \\
.18721 \\
.18724 \\
.18727\end{array}$ & $\begin{array}{l}.003142 \\
.003147 \\
.003153 \\
.003158 \\
.003163\end{array}$ \\
\hline $\begin{array}{r}7,000 \\
20 \\
40 \\
60 \\
80\end{array}$ & $\begin{array}{l}.14558 \\
.14553 \\
.14548 \\
.14543 \\
.14538\end{array}$ & $\begin{array}{l}.18498 \\
.18502 \\
.18506 \\
.18510 \\
.18515\end{array}$ & $\begin{array}{l}.002777 \\
.002784 \\
.002790 \\
.002797 \\
.002804\end{array}$ & $\begin{array}{r}8,300 \\
20 \\
40 \\
60 \\
80\end{array}$ & $\begin{array}{l}.14286 \\
.14283 \\
.14279 \\
.14276 \\
.14272\end{array}$ & $\begin{array}{l}.18730 \\
.18733 \\
.18736 \\
.18739 \\
.18742\end{array}$ & $\begin{array}{l}.003168 \\
.003174 \\
.003179 \\
.003184 \\
.003189\end{array}$ \\
\hline $\begin{array}{r}7,100 \\
20 \\
40 \\
60 \\
80\end{array}$ & $\begin{array}{l}.14533 \\
.14529 \\
.14523 \\
.14518 \\
.14513\end{array}$ & $\begin{array}{l}.18519 \\
.18523 \\
.18527 \\
.18531 \\
.18536\end{array}$ & $\begin{array}{l}.002810 \\
.002817 \\
.002824 \\
.002830 \\
.002837\end{array}$ & $\begin{array}{r}8,400 \\
20 \\
40 \\
60 \\
80\end{array}$ & $\begin{array}{l}.14269 \\
.14265 \\
.14262 \\
.14258 \\
.14255\end{array}$ & $\begin{array}{l}.18745 \\
.18748 \\
.18751 \\
.18753 \\
.18756\end{array}$ & $\begin{array}{l}.003194 \\
.003200 \\
.003205 \\
.003210 \\
.003215\end{array}$ \\
\hline $\begin{array}{r}7,200 \\
20 \\
40 \\
60 \\
80\end{array}$ & $\begin{array}{l}.14509 \\
.14504 \\
.14499 \\
.14494 \\
.14490\end{array}$ & $\begin{array}{l}.18540 \\
.18544 \\
.18548 \\
.18552 \\
.18556\end{array}$ & $\begin{array}{l}.002844 \\
.002850 \\
.002857 \\
.002863 \\
.002870\end{array}$ & $\begin{array}{r}8,500 \\
20 \\
40 \\
60 \\
80\end{array}$ & $\begin{array}{l}.14252 \\
.14249 \\
.14245 \\
.14242 \\
.14239\end{array}$ & $\begin{array}{l}.18759 \\
.18762 \\
.18765 \\
.18767 \\
.18770\end{array}$ & $\begin{array}{l}.003220 \\
.003225 \\
.003230 \\
.003235 \\
.003240\end{array}$ \\
\hline $\begin{array}{r}7,300 \\
20 \\
40 \\
60 \\
80\end{array}$ & $\begin{array}{l}.14485 \\
.14480 \\
.14476 \\
.14471 \\
.14467\end{array}$ & $\begin{array}{l}.18560 \\
.18564 \\
.18568 \\
.18572 \\
.18576\end{array}$ & $\begin{array}{l}.002876 \\
.002883 \\
.002889 \\
.002896 \\
.002902\end{array}$ & $\begin{array}{r}8,600 \\
20 \\
40 \\
60 \\
80\end{array}$ & $\begin{array}{l}.14236 \\
.14233 \\
.14229 \\
.14226 \\
.14223\end{array}$ & $\begin{array}{l}.18773 \\
.18775 \\
.18778 \\
.18781 \\
.18783\end{array}$ & $\begin{array}{l}.003245 \\
.003250 \\
.003255 \\
.003260 \\
.003265\end{array}$ \\
\hline $\begin{array}{r}7,400 \\
20 \\
40 \\
60 \\
80\end{array}$ & $\begin{array}{l}.14462 \\
.14458 \\
.14453 \\
.14449 \\
.14444\end{array}$ & $\begin{array}{l}.18580 \\
.18584 \\
.18588 \\
.18591 \\
.18595\end{array}$ & $\begin{array}{l}.002907 \\
.002915 \\
.002921 \\
.002928 \\
.002934\end{array}$ & $\begin{array}{r}8,700 \\
20 \\
40 \\
60 \\
80\end{array}$ & $\begin{array}{l}.14220 \\
.14217 \\
.14214 \\
.14211 \\
.14208\end{array}$ & $\begin{array}{l}.18786 \\
.18788 \\
.18791 \\
.18794 \\
.18796\end{array}$ & $\begin{array}{l}.003270 \\
.003275 \\
.003280 \\
.003285 \\
.003290\end{array}$ \\
\hline $\begin{array}{r}7,500 \\
20 \\
40 \\
60 \\
80\end{array}$ & $\begin{array}{l}.14440 \\
.14436 \\
.14431 \\
.14427 \\
.14423\end{array}$ & $\begin{array}{l}.18599 \\
.18603 \\
.18606 \\
.18610 \\
.18614\end{array}$ & $\begin{array}{l}.002940 \\
.002946 \\
.002952 \\
.002959 \\
.002965\end{array}$ & $\begin{array}{r}8,800 \\
20 \\
40 \\
60 \\
80\end{array}$ & $\begin{array}{l}.14205 \\
.14202 \\
.14190 \\
.14196 \\
.14193\end{array}$ & $\begin{array}{l}.18799 \\
.18801 \\
.18804 \\
.18806 \\
.18809\end{array}$ & $\begin{array}{r}003295 \\
.003300 \\
.003304 \\
.003309 \\
.003314\end{array}$ \\
\hline $\begin{array}{r}7,600 \\
20 \\
40 \\
60 \\
80\end{array}$ & $\begin{array}{l}.14419 \\
.14415 \\
.14411 \\
.14407 \\
.14402\end{array}$ & $\begin{array}{l}.18617 \\
.18621 \\
.18624 \\
.18628 \\
.18681\end{array}$ & $\begin{array}{l}.002971 \\
.002977 \\
.002983 \\
.002989 \\
.002995\end{array}$ & $\begin{array}{r}8,900 \\
20 \\
40 \\
60 \\
80\end{array}$ & $\begin{array}{l}.14190 \\
.14187 \\
.14184 \\
.14181 \\
.14178\end{array}$ & $\begin{array}{l}.18811 \\
.18814 \\
.18817 \\
.18819 \\
.18821\end{array}$ & $\begin{array}{l}.003319 \\
.003324 \\
.003329 \\
.003334 \\
.003338\end{array}$ \\
\hline
\end{tabular}


TABLE 1.-Table to facilitate the computation of correlated color temperatures-Con.

\begin{tabular}{|c|c|c|c|c|c|c|c|}
\hline $\begin{array}{l}\text { Color } \\
\text { temper- } \\
\text { ature }\end{array}$ & $\omega r L_{r}$ & $\omega g L_{\imath}$ & $\omega b L_{b}$ & $\begin{array}{l}\text { Color } \\
\text { temper- } \\
\text { ature }\end{array}$ & $\omega r L_{r}$ & $\omega g L_{0}$ & $\omega b L_{b}$ \\
\hline $\begin{array}{r}\circ K \\
9,000 \\
20 \\
40 \\
60 \\
80\end{array}$ & $\begin{array}{r}0.14175 \\
.14172 \\
.14169 \\
.14167 \\
.14164\end{array}$ & $\begin{array}{r}0.18824 \\
.18826 \\
.18829 \\
.18831 \\
.18833\end{array}$ & $\begin{array}{r}0.003343 \\
.003348 \\
.003352 \\
.003357 \\
.003361\end{array}$ & $\begin{array}{r}\circ K \\
10,300 \\
20 \\
40 \\
60 \\
80\end{array}$ & $\begin{array}{r}0.14016 \\
.14013 \\
.14011 \\
.14009 \\
.14007\end{array}$ & $\begin{array}{r}0.18956 \\
.18958 \\
.18959 \\
.18961 \\
.18963\end{array}$ & $\begin{array}{r}0.003616 \\
.003620 \\
.003624 \\
.003627 \\
.003631\end{array}$ \\
\hline $\begin{array}{r}9,100 \\
20 \\
40 \\
60 \\
80\end{array}$ & $\begin{array}{l}.14161 \\
.14158 \\
.14156 \\
.14153 \\
.14150\end{array}$ & $\begin{array}{l}.18835 \\
.18837 \\
.18840 \\
.18842 \\
.18844\end{array}$ & $\begin{array}{l}.003364 \\
.003370 \\
.003375 \\
.003380 \\
.003384\end{array}$ & $\begin{array}{r}10,400 \\
20 \\
40 \\
60 \\
80\end{array}$ & $\begin{array}{l}.14005 \\
.14003 \\
.14001 \\
.13999 \\
.13997\end{array}$ & $\begin{array}{l}.18965 \\
.18966 \\
.18968 \\
.18970 \\
.18971\end{array}$ & $\begin{array}{l}.003634 \\
.003638 \\
.003641 \\
.003645 \\
.003649\end{array}$ \\
\hline $\begin{array}{r}9,200 \\
20 \\
40 \\
60 \\
80\end{array}$ & $\begin{array}{l}.14148 \\
.14145 \\
.14142 \\
.14139 \\
.14137\end{array}$ & $\begin{array}{l}.18846 \\
.18849 \\
.18851 \\
.18853 \\
.18855\end{array}$ & $\begin{array}{l}.003389 \\
.003393 \\
.003398 \\
.003402 \\
.003406\end{array}$ & $\begin{array}{r}10,500 \\
20 \\
40 \\
60 \\
80\end{array}$ & $\begin{array}{l}.13995 \\
.13993 \\
.13991 \\
.13989 \\
.13987\end{array}$ & $\begin{array}{l}.18973 \\
.18975 \\
.18976 \\
.18978 \\
.18980\end{array}$ & $\begin{array}{l}.003652 \\
.003656 \\
.003659 \\
.003663 \\
.003666\end{array}$ \\
\hline $\begin{array}{r}9,300 \\
20 \\
40 \\
60 \\
80\end{array}$ & $\begin{array}{l}.14134 \\
.14131 \\
.14129 \\
.14126 \\
.14123\end{array}$ & $\begin{array}{l}.18857 \\
.18860 \\
.18862 \\
.18864 \\
.18866\end{array}$ & $\begin{array}{l}.003411 \\
.003415 \\
.003420 \\
.003424 \\
.003429\end{array}$ & $\begin{array}{r}10,600 \\
20 \\
40 \\
60 \\
80\end{array}$ & $\begin{array}{l}.13985 \\
.13983 \\
.13981 \\
.13979 \\
.13977\end{array}$ & $\begin{array}{r}.18981 \\
.18983 \\
.18985 \\
.18986 \\
.18988\end{array}$ & $\begin{array}{l}.003670 \\
.003673 \\
.003677 \\
.003680 \\
.003684\end{array}$ \\
\hline $\begin{array}{r}9,400 \\
20 \\
40 \\
60 \\
80\end{array}$ & $\begin{array}{l}.14121 \\
.14118 \\
.14116 \\
.14113 \\
.14110\end{array}$ & $\begin{array}{l}.18868 \\
.18871 \\
.18873 \\
.18875 \\
.18877\end{array}$ & $\begin{array}{l}.003433 \\
.003438 \\
.003442 \\
.003446 \\
.003451\end{array}$ & $\begin{array}{r}10,700 \\
20 \\
40 \\
60 \\
80\end{array}$ & $\begin{array}{l}.13975 \\
.13973 \\
.13971 \\
.13969 \\
.13967\end{array}$ & $\begin{array}{l}.18990 \\
.18991 \\
.18993 \\
.18994 \\
.18996\end{array}$ & $\begin{array}{l}.003688 \\
.003691 \\
.003695 \\
.003698 \\
.003702\end{array}$ \\
\hline $\begin{array}{r}9,500 \\
20 \\
40 \\
60 \\
80\end{array}$ & $\begin{array}{l}.14108 \\
.14105 \\
.14103 \\
.14100 \\
.14098\end{array}$ & $\begin{array}{l}.18879 \\
.18881 \\
.18883 \\
.18885 \\
.18887\end{array}$ & $\begin{array}{l}.003455 \\
.003459 \\
.003464 \\
.003468 \\
.003472\end{array}$ & $\begin{array}{r}10,800 \\
20 \\
40 \\
60 \\
80\end{array}$ & $\begin{array}{l}.13965 \\
.13963 \\
.13961 \\
.13959 \\
.13957\end{array}$ & $\begin{array}{l}.18998 \\
.18999 \\
.19001 \\
.19003 \\
.19004\end{array}$ & $\begin{array}{l}.003705 \\
.003709 \\
.003712 \\
.003716 \\
.003719\end{array}$ \\
\hline $\begin{array}{r}9,600 \\
20 \\
40 \\
60 \\
80\end{array}$ & $\begin{array}{l}.14095 \\
.14093 \\
.14090 \\
.14088 \\
.14085\end{array}$ & $\begin{array}{l}.18889 \\
.18891 \\
.18893 \\
.18896 \\
.18898\end{array}$ & $\begin{array}{l}.003477 \\
.003481 \\
.003486 \\
.003490 \\
.003494\end{array}$ & $\begin{array}{r}10,900 \\
20 \\
40 \\
60 \\
80\end{array}$ & $\begin{array}{r}.13955 \\
.13953 \\
.13951 \\
.13949 \\
.13947\end{array}$ & $\begin{array}{l}.19006 \\
.19008 \\
.19009 \\
.19011 \\
.19012\end{array}$ & $\begin{array}{l}.003723 \\
.003726 \\
.003730 \\
.003733 \\
.003737\end{array}$ \\
\hline $\begin{array}{r}9,700 \\
20 \\
40 \\
60 \\
80\end{array}$ & $\begin{array}{l}.14083 \\
.14081 \\
.14078 \\
.14076 \\
.14073\end{array}$ & $\begin{array}{l}.18900 \\
.18902 \\
.18904 \\
.18906 \\
.18908\end{array}$ & $\begin{array}{l}.003498 \\
.003503 \\
.003507 \\
.003511 \\
.003515\end{array}$ & $\begin{array}{r}11,000 \\
20 \\
40 \\
60 \\
80\end{array}$ & $\begin{array}{l}.13945 \\
.13943 \\
.13941 \\
.13939 \\
.13938\end{array}$ & $\begin{array}{l}.19014 \\
.19015 \\
.19017 \\
.19019 \\
.19020\end{array}$ & $\begin{array}{l}.003740 \\
.003744 \\
.003747 \\
.003750 \\
.003754\end{array}$ \\
\hline $\begin{array}{r}9,800 \\
20 \\
40 \\
60 \\
80\end{array}$ & $\begin{array}{l}.14071 \\
.14069 \\
.14066 \\
.14064 \\
.14062\end{array}$ & $\begin{array}{l}.18910 \\
.18912 \\
.18914 \\
.18916 \\
.18917\end{array}$ & $\begin{array}{l}.003519 \\
.003524 \\
.003528 \\
.003532 \\
.003536\end{array}$ & $\begin{array}{r}11,100 \\
20 \\
40 \\
60 \\
80\end{array}$ & $\begin{array}{l}.13936 \\
.13934 \\
.13932 \\
.13930 \\
.13928\end{array}$ & $\begin{array}{l}.19022 \\
.19023 \\
.19025 \\
.19026 \\
.19028\end{array}$ & $\begin{array}{l}.003757 \\
.003761 \\
.003764 \\
.003768 \\
.003771\end{array}$ \\
\hline $\begin{array}{r}9,900 \\
20 \\
40 \\
60 \\
80\end{array}$ & $\begin{array}{l}.14059 \\
.14057 \\
.14055 \\
.14052 \\
.14050\end{array}$ & $\begin{array}{l}.18919 \\
.18921 \\
.18923 \\
.18925 \\
.18927\end{array}$ & $\begin{array}{l}.003540 \\
.003544 \\
.003548 \\
.003552 \\
.003556\end{array}$ & $\begin{array}{r}11,200 \\
20 \\
40 \\
60 \\
80\end{array}$ & $\begin{array}{l}.13927 \\
.13925 \\
.13923 \\
.13921 \\
.13920\end{array}$ & $\begin{array}{l}.19029 \\
.19030 \\
.19032 \\
.19033 \\
.19035\end{array}$ & $\begin{array}{l}.003774 \\
.003778 \\
.003781 \\
.003784 \\
.003788\end{array}$ \\
\hline $\begin{array}{r}10,000 \\
20 \\
40 \\
60 \\
80\end{array}$ & $\begin{array}{l}.14048 \\
.14046 \\
.14043 \\
.14041 \\
.14039\end{array}$ & $\begin{array}{l}.18929 \\
.18931 \\
.18933 \\
.18935 \\
.18936\end{array}$ & $\begin{array}{l}.003560 \\
.003564 \\
.003568 \\
.003572 \\
.003576\end{array}$ & $\begin{array}{r}11,300 \\
20 \\
40 \\
60 \\
80\end{array}$ & $\begin{array}{l}.13918 \\
.13916 \\
.13914 \\
.13913 \\
.13911\end{array}$ & $\begin{array}{l}.19036 \\
.19038 \\
.19039 \\
.19040 \\
.19042\end{array}$ & $\begin{array}{l}.003791 \\
.003794 \\
.003798 \\
.003801 \\
.003504\end{array}$ \\
\hline $\begin{array}{r}10,100 \\
20 \\
40 \\
60 \\
80\end{array}$ & $\begin{array}{l}.14037 \\
.14035 \\
.14033 \\
.14030 \\
.14028\end{array}$ & $\begin{array}{l}.18938 \\
.18940 \\
.18942 \\
.18944 \\
.18945\end{array}$ & $\begin{array}{l}.003579 \\
.003583 \\
.003587 \\
.003591 \\
.003595\end{array}$ & $\begin{array}{r}11,400 \\
20 \\
40 \\
60 \\
80\end{array}$ & $\begin{array}{l}.13909 \\
.13908 \\
.13906 \\
.13904 \\
.13903\end{array}$ & $\begin{array}{l}.19043 \\
.19045 \\
.19046 \\
.19047 \\
.19049\end{array}$ & $\begin{array}{l}.003807 \\
.003810 \\
.003814 \\
.003817 \\
.003820\end{array}$ \\
\hline $\begin{array}{r}10,200 \\
20 \\
40 \\
60 \\
80\end{array}$ & $\begin{array}{l}.14026 \\
.14024 \\
.14022 \\
.14020 \\
.14018\end{array}$ & $\begin{array}{l}.18947 \\
.18949 \\
.18951 \\
.18952 \\
.18954\end{array}$ & $\begin{array}{l}.003598 \\
.003602 \\
.003606 \\
.003609 \\
.003613\end{array}$ & $\begin{array}{r}11,500 \\
20 \\
40 \\
60 \\
80\end{array}$ & $\begin{array}{l}.13901 \\
.13899 \\
.13898 \\
.13896 \\
.13894\end{array}$ & $\begin{array}{l}.19050 \\
.19051 \\
.19053 \\
.19054 \\
.19055\end{array}$ & $\begin{array}{l}.003823 \\
.003826 \\
.003829 \\
.003832 \\
.003835\end{array}$ \\
\hline
\end{tabular}


TABLE 1.-Table to facilitate the computation of correlated color temperatures-Con.

\begin{tabular}{|c|c|c|c|c|c|c|c|}
\hline $\begin{array}{l}\text { Color } \\
\text { temper- } \\
\text { ature }\end{array}$ & $\omega r L_{r}$ & $\omega g L_{g}$ & $\omega b L_{b}$ & $\begin{array}{l}\text { Color } \\
\text { temper- } \\
\text { ature }\end{array}$ & $\omega r L_{r}$ & $\omega g L$ & $\omega b L_{b}$ \\
\hline $\begin{array}{r}\circ K \\
11,600 \\
20 \\
40 \\
60 \\
80\end{array}$ & $\begin{array}{r}0.13893 \\
.13891 \\
.13889 \\
.13888 \\
.13886\end{array}$ & $\begin{array}{r}0.19057 \\
.19058 \\
.19059 \\
.19060 \\
.19062\end{array}$ & $\begin{array}{r}0.003838 \\
.003841 \\
.003844 \\
.003847 \\
.003850\end{array}$ & $\begin{array}{r}\circ K \\
15,000 \\
100 \\
200 \\
300 \\
400\end{array}$ & $\begin{array}{r}0.13684 \\
.13680 \\
.13675 \\
.13671 \\
.13666\end{array}$ & $\begin{array}{r}0.19223 \\
.19227 \\
.19231 \\
.19234 \\
.19238\end{array}$ & $\begin{array}{r}0.004248 \\
.004257 \\
.004267 \\
.004276 \\
.004284\end{array}$ \\
\hline $\begin{array}{r}11,700 \\
20 \\
40 \\
60 \\
80\end{array}$ & $\begin{array}{l}.13884 \\
.13883 \\
.13881 \\
.13880 \\
.13378\end{array}$ & $\begin{array}{l}.19063 \\
.19064 \\
.19066 \\
.19067 \\
.19068\end{array}$ & $\begin{array}{l}.003853 \\
.003856 \\
.0038 \mathrm{r} 0 \\
.003863 \\
.003866\end{array}$ & $\begin{array}{r}15,500 \\
600 \\
700 \\
800 \\
800\end{array}$ & $\begin{array}{l}.13662 \\
.13658 \\
.13653 \\
.13649 \\
.13645\end{array}$ & $\begin{array}{l}.19241 \\
.19245 \\
.19248 \\
.19251 \\
.19255\end{array}$ & $\begin{array}{l}.004293 \\
.004302 \\
.004311 \\
.004318 \\
.004327\end{array}$ \\
\hline $\begin{array}{r}11,800 \\
20 \\
40 \\
60 \\
80\end{array}$ & $\begin{array}{l}.13877 \\
.13875 \\
.13873 \\
.13872 \\
.13870\end{array}$ & $\begin{array}{l}.19089 \\
.19071 \\
.19072 \\
.19073 \\
.19074\end{array}$ & $\begin{array}{l}.003869 \\
.003872 \\
.003874 \\
.003877 \\
.003880\end{array}$ & $\begin{array}{r}16,000 \\
100 \\
200 \\
300 \\
400\end{array}$ & $\begin{array}{l}.13641 \\
.13637 \\
.13633 \\
.13630 \\
.13626\end{array}$ & $\begin{array}{l}.19258 \\
.19261 \\
.19264 \\
.19267 \\
.19270\end{array}$ & $\begin{array}{l}.004337 \\
.004343 \\
.004352 \\
.004360 \\
.004368\end{array}$ \\
\hline $\begin{array}{r}11,900 \\
20 \\
40 \\
60 \\
80\end{array}$ & $\begin{array}{l}.13869 \\
.13867 \\
.13866 \\
.13864 \\
.13862\end{array}$ & $\begin{array}{l}.19076 \\
.19077 \\
.19078 \\
.19079 \\
.19081\end{array}$ & $\begin{array}{l}.003883 \\
.003886 \\
.003889 \\
.003892 \\
.003895\end{array}$ & $\begin{array}{r}16,500 \\
600 \\
700 \\
800 \\
900\end{array}$ & $\begin{array}{l}.13622 \\
.13618 \\
.13615 \\
.13611 \\
.13608\end{array}$ & $\begin{array}{l}.19273 \\
.19276 \\
.19279 \\
.19282 \\
.19285\end{array}$ & $\begin{array}{l}.004377 \\
.004384 \\
.004392 \\
.004400 \\
.004407\end{array}$ \\
\hline $\begin{array}{r}12,000 \\
100 \\
200 \\
300 \\
400\end{array}$ & $\begin{array}{l}.13861 \\
.13854 \\
.13847 \\
.13839 \\
.13832\end{array}$ & $\begin{array}{l}.19082 \\
.19088 \\
.19093 \\
.19099 \\
.19105\end{array}$ & $\begin{array}{l}.003898 \\
.003911 \\
.003925 \\
.003938 \\
.003952\end{array}$ & $\begin{array}{r}17,000 \\
100 \\
200 \\
300 \\
400\end{array}$ & $\begin{array}{l}.13604 \\
.13601 \\
.13597 \\
.13594 \\
.13591\end{array}$ & $\begin{array}{l}.19288 \\
.19290 \\
.19203 \\
.19296 \\
.19298\end{array}$ & $\begin{array}{l}.004414 \\
.004422 \\
.004429 \\
.004437 \\
.004444\end{array}$ \\
\hline $\begin{array}{r}12,500 \\
600 \\
700 \\
800 \\
900\end{array}$ & $\begin{array}{l}.13825 \\
.13819 \\
.13812 \\
.13805 \\
.13799\end{array}$ & $\begin{array}{l}.19111 \\
.19116 \\
.19122 \\
.19127 \\
.19132\end{array}$ & $\begin{array}{l}.003966 \\
.003978 \\
.003992 \\
.004004 \\
.004017\end{array}$ & $\begin{array}{r}17,500 \\
600 \\
700 \\
800 \\
900\end{array}$ & $\begin{array}{l}.13587 \\
.13584 \\
.13581 \\
.13578 \\
.13575\end{array}$ & $\begin{array}{l}.19301 \\
.19303 \\
.19306 \\
.19308 \\
.19311\end{array}$ & $\begin{array}{l}.004451 \\
.004458 \\
.004464 \\
.004471 \\
.004478\end{array}$ \\
\hline $\begin{array}{r}13,000 \\
100 \\
200 \\
300 \\
400\end{array}$ & $\begin{array}{l}.13792 \\
.13786 \\
.13780 \\
.13774 \\
.13768\end{array}$ & $\begin{array}{r}.19137 \\
.19142 \\
.19147 \\
.19152 \\
.19157\end{array}$ & $\begin{array}{l}.004029 \\
.004042 \\
.004054 \\
.004067 \\
.004078\end{array}$ & $\begin{array}{r}18,000 \\
100 \\
200 \\
300 \\
400\end{array}$ & $\begin{array}{l}.13572 \\
.13569 \\
.13566 \\
.13563 \\
.13561\end{array}$ & $\begin{array}{l}.19313 \\
.19315 \\
.19318 \\
.19320 \\
.19322\end{array}$ & $\begin{array}{l}.004484 \\
.004491 \\
.004497 \\
.004503 \\
.004510\end{array}$ \\
\hline $\begin{array}{r}13,500 \\
600 \\
700 \\
800 \\
900\end{array}$ & $\begin{array}{l}.13762 \\
.13756 \\
.13751 \\
.13745 \\
.13740\end{array}$ & $\begin{array}{l}.19162 \\
.19166 \\
.19171 \\
.19175 \\
.19180\end{array}$ & $\begin{array}{l}.004090 \\
.004102 \\
.004113 \\
.004124 \\
.004135\end{array}$ & $\begin{array}{r}18,500 \\
600 \\
700 \\
800 \\
900\end{array}$ & $\begin{array}{l}.13558 \\
.13555 \\
.13553 \\
.13550 \\
.13547\end{array}$ & $\begin{array}{l}.19324 \\
.19326 \\
.19328 \\
.19330 \\
.19332\end{array}$ & $\begin{array}{l}.004516 \\
.004522 \\
.004528 \\
.004533 \\
.004540\end{array}$ \\
\hline $\begin{array}{r}14,000 \\
100 \\
200 \\
300 \\
400\end{array}$ & $\begin{array}{r}13734 \\
.13729 \\
.13724 \\
.13718 \\
13713\end{array}$ & $\begin{array}{r}.19184 \\
.19188 \\
.19192 \\
.19196 \\
.19200\end{array}$ & $\begin{array}{l}.004146 \\
.004157 \\
.004167 \\
.004177\end{array}$ & $\begin{array}{r}19,000 \\
100 \\
200 \\
300 \\
400\end{array}$ & $\begin{array}{l}.13545 \\
.13542 \\
.13540 \\
.13537 \\
.13535\end{array}$ & $\begin{array}{l}.19334 \\
.19336 \\
.19338 \\
.19340 \\
.19342\end{array}$ & $\begin{array}{l}.004546 \\
.004552 \\
.004556 \\
.004563 \\
.004568\end{array}$ \\
\hline $\begin{array}{r}14,500 \\
600 \\
700 \\
800 \\
900\end{array}$ & $\begin{array}{l}.13708 \\
.13703 \\
.13699 \\
.13694 \\
.13689\end{array}$ & $\begin{array}{l}.19204 \\
.19208 \\
.19212 \\
.19216 \\
.19220\end{array}$ & $\begin{array}{r}.004188 \\
.004198 \\
.004208 \\
.004218 \\
.004228 \\
.004238\end{array}$ & $\begin{array}{r}19,500 \\
600 \\
700 \\
800 \\
900 \\
20,000\end{array}$ & $\begin{array}{l}.13532 \\
.13530 \\
.13527 \\
.13525 \\
.13522 \\
.13520\end{array}$ & $\begin{array}{l}.19344 \\
.19346 \\
.19348 \\
.19349 \\
.19351 \\
.19353\end{array}$ & $\begin{array}{l}.004573 \\
.004579 \\
.004584 \\
.004590 \\
.004595 \\
.004600\end{array}$ \\
\hline
\end{tabular}

\section{COMPUTATION OF THE CORRELATED COLOR TEMPERATURE}

To make the directions more specific the correlated color temperature of sunlight outside the earth's atmosphere will be computed. Using Abbot's 1917 data (4, Table 1) and following the form given in (4, fig. 12) the values of $r, g$, and $b$ were calculated. 
Thus

$$
\begin{array}{r}
r=0.29883 ; L_{r}=0.45014 ; r L_{r}=0.134515 \\
g=0.31261 ; L_{g}=0.54417 ; g L_{g}=.170113 \\
b=0.38856 ; L_{b}=0.00569 ; b L_{b}=.002211 \\
r L_{r}+g L_{g}+b L_{b}=.306839 \\
\omega=\frac{0.333333}{0.306839}=1.08635 \\
\omega r L_{r}=.14613 \\
\omega g L_{g}=.18480 \\
\omega b L_{b}=.002402
\end{array}
$$

(Check:- $\omega r L_{\tau}+\omega g L_{g}+\omega b L_{b}=.33333$ )

Seeking these values of $\omega r L_{r}, \omega g L_{0}$, and $\omega b L_{b}$ in the respective columns of Table 1 we obtain the following component temperatures:

For

\begin{tabular}{|c|c|c|c|c|c|c|}
\hline \multirow{3}{*}{ Energy distribution used in computation } & \multicolumn{6}{|c|}{ Correlated color temperatures } \\
\hline & \multicolumn{3}{|c|}{ Computed values } & \multicolumn{3}{|c|}{ Observed values } \\
\hline & $\begin{array}{l}\text { Refer- } \\
\text { ence }\end{array}$ & $\begin{array}{c}\lambda_{e} \\
\text { method }\end{array}$ & $\begin{array}{l}\text { Present } \\
\text { method }\end{array}$ & $\begin{array}{l}\text { Re er- } \\
\text { ence }\end{array}$ & Time observed & Value \\
\hline \multirow{2}{*}{$\begin{array}{l}\text { Mean noon sun } \\
\text { Mean noon sun summer solistice. } \\
\text { Mean noon sun winter solstice... } \\
\text { Sun outside earth's atmosphere. } \\
\text { Abbot-Priest sun }\end{array}$} & $\begin{array}{l}\text { (4) } \ldots \text { (4) }-\ldots \\
\text { (4) } \ldots \ldots\end{array}$ & \multirow{2}{*}{$\begin{array}{l}\circ K . \\
5,578 \\
5,918 \\
5,275 \\
6,724 \\
5,323\end{array}$} & \multirow{2}{*}{$\begin{array}{l}{ }^{\circ} K \\
5,394 \\
5,740 \\
5,077 \\
6,565 \\
5,229\end{array}$} & $\begin{array}{l}(12) \ldots \\
(12) \ldots \\
(12) \ldots\end{array}$ & $\begin{array}{l}\text { 2/20/20 noon } \\
\text { 6/26/22 12:30 p. m- } \\
1 / 24 / 251: 30 \mathrm{p} . \mathrm{m}\end{array}$ & $\begin{array}{l}{ }^{\circ} K \\
15,200 \\
15,300 \\
15,212\end{array}$ \\
\hline & (12) & & & $(12) \ldots$ & & \multirow[t]{2}{*}{$\begin{array}{l}5,323 \\
5,216\end{array}$} \\
\hline \multirow{4}{*}{$\begin{array}{l}\text { Equal energy spectrum } \\
\text { Acetylene flame } \\
\text { B. S. lamp No. } 1717 \\
\text { Blue glass R4-28 (source at } 2,077^{\circ} \mathrm{K} \text {.) } \\
\text { Blue glass R4-28 (source at } 2,080^{\circ} \mathrm{K} \text {.) } \\
\text { Blue glass R4-28 (source at } 2,360^{\circ} \mathrm{K} \text {.) }\end{array}$} & & 5,212 & 5,357 & & & \\
\hline & $\left\{\begin{array}{l}(4) \ldots . . . \\
11) \ldots\end{array}\right.$ & $\begin{array}{l}2,388 \\
2,848 \\
2,358\end{array}$ & $\begin{array}{l}2,413 \\
2,860 \\
2,386\end{array}$ & $\begin{array}{l}(4) \ldots \\
(11) \ldots \\
-\ldots\end{array}$ & & $\begin{array}{r}2 \\
2,420 \\
2,848 \\
\end{array}$ \\
\hline & & & 2,389 & & & 32,390 \\
\hline & & & 2,779 & & & \\
\hline
\end{tabular}

$$
\begin{array}{cc}
{ }^{\circ} K \\
\omega r L_{r}, & 6,790 \\
\omega g L_{g}, & 6,916 \\
\omega b L_{b}, & 5,989
\end{array}
$$

Correlated color temperature (mean) 6,565

Table 2 gives for a number of sources a comparison of the values calculated by the $\lambda_{c}$ method, the present method and also what may be designated as observed magnitudes.

TABLE 2.-Comparison of correlated color temperatures obtained by computation and observation

1 These values are isolated measurements, and are not necessarily representative of "mean" atmospheric conditions. Furthermore, the days on which these measurements were made are not wholly consistent with the dates assumed for the computation.

2 For a discussion of the color of the acetylene flame, see B. S. Misc. Pub. No. 114, Secs. III, 4; V, 6, (a); VIII, 6.

${ }_{3}$ Quoting from National Physical Laboratory's Report dated January, 1930 (International Comparison of the Transmissions of Four Blue Glasses): "It was found by color matching experiments at the National Physical Laboratory that the glasses would change the light from a tungsten lamp operating at a color temperature of $2,080^{\circ} \mathrm{K}$. to light having a color temperature of $2,390^{\circ} \mathrm{K}$,

- Quoting again the National Physical Laboratory Report: "By calculation it was then found that the same glasses would change the light from a tungsten lamp operated at a color temperature of $2,360^{\circ} \mathrm{K}$. to light having a color temperature of $2,770^{\circ} \mathrm{K}$. which corresponds to about 13 lumens per watt for a gas filled lamp." 
TABLE 3.-Correlated color temperature data for well-known energy distributions ${ }^{1}$

\begin{tabular}{|c|c|c|c|c|}
\hline Source of energy & $\begin{array}{l}\text { Corre- } \\
\text { lated } \\
\text { color } \\
\text { temper- } \\
\text { ture } \\
\left(\theta_{i}\right)\end{array}$ & $\begin{array}{c}\text { Mean } \\
\text { devia- } \\
\text { tion } \\
(\sigma)\end{array}$ & $\begin{array}{c}\text { Sensa- } \\
\text { tion } \\
\text { steps } \\
\frac{\sigma}{\delta}\end{array}$ & $\begin{array}{c}\text { Conj. } \\
\text { wave } \\
\text { length } \\
\left(\lambda_{\mathbf{j}}\right)\end{array}$ \\
\hline $\begin{array}{l}\text { Mean nocn sun } \\
\text { Mean noon sun, summer solstice } \\
\text { Mean noon sun, winter solstice } \\
\text { Abbot-Priest sun } \\
\text { A cetylene, from Coblentz' data } \\
\text { Lamp } 1717 \text { (bib. ref. 11), from Coblentz data } \\
\text { Equal energy spectrum }\end{array}$ & $\begin{array}{c}{ }^{\circ} K . \\
\mathbf{5}, 394 \\
\mathbf{5}, 740 \\
5,077 \\
5,229 \\
2,413 \\
2,860 \\
5,357\end{array}$ & $\begin{array}{r}{ }^{\circ} K . \\
347 \\
365 \\
345 \\
219 \\
38 \\
18 \\
291\end{array}$ & $\begin{array}{r}11.9 \\
11.1 \\
13.4 \\
8.0 \\
6.6 \\
2.2 \\
10.1\end{array}$ & $\begin{array}{r}\mu \\
566.8 \\
566.3 \\
567.6 \\
567.0 \\
2-580.5 \\
576.7 \\
-565.7\end{array}$ \\
\hline
\end{tabular}

1 The specific energy distributions for which these values apply may be found in B. S. Misc. Pub. No. 114 (4) - the noon sun data in Table 1, the acetylene data in Table 3, Abbot-Priest sun in fig. 3, and Iamp 1717 in fig. 7.

2 The negative sign indicates that light of the conjunctive wave length must be added to the stimulus in question in order to match the Planckian color.

\section{CALCULATION OF COLOR DEPARTURE IN TERMS OF SENSATION STEPS}

Within any small region near the Planckian locus the ratio of the color departure to the mean deviation should be constant. Since, as is well known, the perceptibility of a unit increment in color temperature decreases rapidly with increase in temperature, it is desirable to express mean deviation in "sensation" steps rather than in degrees of temperature.

Priest (2) has shown that between $3,000^{\circ}$ and $20,000^{\circ} \mathrm{K}$. the probable error of a single observation in color matching is between 0.1 and $0.2 \mathrm{~m} \mu$ as expressed on the $\lambda_{c}$ scale, without showing any systematic variation with color temperature. The reasonable assumption is made that the number of degrees just perceptibly different in color (a sensation step) at any color temperature, corresponds to a constant difference in $\lambda_{c}$ values.

An attempt made to obtain a more convenient method resulted in the discovery that the number of degrees represented by a constant difference in $\lambda_{c}$ over the range $2,000^{\circ}$ to $24,000^{\circ} \mathrm{K}$. varies quite closely as the square of the color temperature. ${ }^{4}$

Hence, a "sensation step" in degrees along the Planckian locus for any color temperature, may be calculated approximately from the relation

where:

$$
\delta=K \theta^{2}
$$

$\theta=a$ sensation step measured in degrees, along the Planckian locus.

$K=$ a constant evaluated by experiment.

$\theta=$ the color temperature in degrees Kelvin.

At $2,360^{\circ} \mathrm{K}$., a point much used in color matching of incandescent electric lamps, a difference of $5^{\circ}$ or $6^{\circ}$ in color temperature of the two fields of the photometer is roughly the magnitude of the "least perceptible difference" in color under the best observing conditions (9). Substituting $5.6^{\circ}$ for $\delta$ and $2,360^{\circ}$ for $\theta$ gives $K=1 \times 10^{-6} .5$

\footnotetext{
4 Veriflcation of this statement may be obtained by utilizing the $\lambda_{0}$ data in Tables 1, 2, 3, and 4 of the A ppendix to Priest's paper (2)

A value of $K=1 \times 10^{-6}$ corresponds to $\delta=0.07 \mathrm{~m} \mu$ on the $\lambda_{c}$ scale. Footnote 15 of Priest's paper (2), gives the values 0.1 and $0.2 \mathrm{~m} \mu$ as representing on the $\lambda_{\epsilon}$ scale the probable error of a single observation, stating however: "These values apply only to certain experimental conditions. A bsolutely smaller probable errors can probably be obtained under improved conditions of observation."
} 
The above relation permits the calculation of $\delta$ (the magnitude of one "sensation step") in degrees along the Planckian locus at any temperature. The ratio $\frac{\sigma}{\delta}$, then, expresses mean deviation in "sensation steps" whose size must necessarily be constant for sufficiently restricted regions near the Planckian locus. Whether the size of these "sensation steps" varies appreciably with temperature is not known; however, we assume in what follows that their size may be considered constant within the limits $1,600^{\circ}$ to $20,000^{\circ} \mathrm{K}$. Whether this assumption proves to be wholly justified or not is of little practical importance because accurate comparisons in terms of "sensation steps" are of interest chiefly within restricted ranges of correlated color temperature.

These steps are considerably smaller than the satron as defined by Priest ( 4 ; p. 121). For example, it is estimated that AbbotPriest sunlight is about 1.6 satrons from $5,229^{\circ} \mathrm{K}$., the correlated color temperature of Abbot-Priest sunlight. This compared with 8 steps $\left(\frac{\sigma}{\delta}\right)$ as given in Table 3 shows that a satron at this point is approximately equivalent to five of the units used in this paper for describing the magnitude of the color difference. While these units may seem quite small compared with the satron it should be pointed out that an absolutely smaller satron locus could be obtained under improved observing conditions. For the purpose used in this paper the size of the unit is of little consequence.

In the example of the correlated color temperature of sunlight outside the earth's atmosphere, given at the end of the previous section, we had obtained component temperatures of $6,790^{\circ}, 6,916^{\circ}$, and $5,989^{\circ} \mathrm{K}$., respectively. The average of these is $6,565^{\circ} \mathrm{K}$. Taking the mean of the deviations of the component temperatures from the average we have

$$
\sigma=\frac{\left(6,790^{\circ}-6,565^{\circ}\right)+\left(6,916^{\circ}-6,565^{\circ}\right)+\left(6,565^{\circ}-5,989^{\circ}\right)}{3}=384^{\circ}
$$

To determine the divergence of the color match in least perceptible differences in color, we find from the relation

That

$$
\delta=K \theta^{2}
$$

$$
\begin{gathered}
\left.\delta=\frac{6,565^{2}}{1,000,000}=43.1^{\circ} \text { (a sensation step at } 6,565^{\circ} \mathrm{K}\right) . \\
\frac{\sigma}{\delta}=\frac{384}{43.1}=8.9 \begin{array}{l}
\text { sensation steps (a measure of the color departure } \\
\text { of sunlight outside the earth's atmosphere from the } \\
\text { Planckian radiator at } 6,565^{\circ} \mathrm{K} \text { ) } .
\end{array}
\end{gathered}
$$

Table 3 gives for a number of sources the correlated color temperatures, the values of mean deviation $\sigma$, their color departure $\frac{\sigma}{\delta}$ in sensation steps, together with the conjunctive wave length $\lambda_{j}$. 


\section{DETERMINATION OF THE CONJUNCTIVE WAVE LENGTH}

In homo-hetero-analysis, a color stimulus is specified by giving its dominant wave length and its purity. The dominant wave length may be computed or it may be found graphically from the trilinear diagram by drawing a straight line through the center of the color triangle and that point representing the color of the stimulus to be specified and extending it until it cuts the spectrum locus.

In the present case, it is desired to specify a non-Planckian stimulus, not with reference to a fixed "neutral" standard, but rather with reference to a particular Planckian distribution as standard; that is, we wish to find the homogeneous stimulus which, when added to or subtracted from (as the case may be) the non-Planckian stimulus will produce a perfect color match. This is found graphically from the familiar trilinear diagram as the intersection of the spectrum locus and a straight line passing through the points representing the two energy distributions. It would appear that this color difference between two light qualities, neither of which is the "neutral" standard, has been so seldom used that it has not been given a name to distinguish it from dominant wave length. The term conjunctive wave length $\left(\lambda_{j}\right)$ is used in the present discussion. ${ }^{6}$ Conjunctive wave lengths for several non-Planckian colors are given in Table 3 and are considered in more detail below.

\section{CHARACTERISTICS OF CORRELATED COLOR TEMPERATURES}

\section{COLOR DEPARTURE AND THE ISOTEMPERATURE LINE}

The work involved in carrying out the computations necessary to make a large-scale graph showing the system of isotemperature lines on the trilinear diagram is so great that a less laborious procedure illustrating the characteristics of the method was adopted. These data are given in Tables 4 and 5 . A group of colors representing hypothetical energy distributions were selected as follows: The $r$, $g$, and $b$ coordinates for $2,000^{\circ}, 3,000^{\circ}$, and $10,000^{\circ} \mathrm{K}$. were changed to represent non-Planckian colors by adding an increment, 0.0070 , to the $g$ coordinate and subtracting a similar value from the $b$ coordinate in each case. The $r$ coordinate was, of course, unchanged since $r, g$, and $b$ sum to unity by definition. In this way colors were selected which fall on a curve approximately parallel to the Planckian locus on the spectrum-locus side. Correlated color temperatures were then computed for the colors represented by these new $r, g$, and $b$ coordinates. The values were $2,011^{\circ}, 3,053^{\circ}, 6,359^{\circ}$, and $11,587^{\circ} \mathrm{K}$. The $r, g$, and $b$ coordinates were then obtained by interpolation from a previously published table (4, Table 11) for each of these color temperatures. The differences were obtained between the

\footnotetext{
- The term conjunctive wave length need not be restricted to the particular use made of it in this paper, but can be employer in all cases where an expression of the chromaticity difference between any two stimuli (neither of which is the "neutral" standard) is desired in terms of the addition (or subtraction) of a certain amount of homogenous light to (or from) ne of the stimuli so that it will match the other. It may frequently happen that a line passing through the 2 points $\left(r_{1}, g_{1}, b_{1} ; r_{2}, g_{2}\right.$ and $\left.b_{2}\right)$ representing the given stimuli will intersect the spectrum locus at 2 points, then each stimulus may be said to nave 2 conjunctive wave lengths with respect to the other. However, this condition will not be found in any case where a non-Planckian stimulus is correlated by the present method with a Planckian stimulus. Conjunctive wave length approaches dominant wave length as a limit as the trilinear coordinates $\left(r_{1}, g_{1}, b_{1}\right.$, or $\left.r_{2}, g_{2}, b_{2}\right)$ of one of the colors approach those corresponding to the "neutral" stimulus for which $r=g=b=1 / 3$.
} 
respective $r$ coordinates, $g$ coordinates, and $b$ coordinates of each of the non-Planckian colors and those of the corresponding correlated color temperatures. By adding 0.5 1, 2, 4, and in two instances, 8 times these differences, according to sign, to the Planckian coordinates new $r, g$, and $b$ coordinates were obtained for a series of colors which plot on a straight line; that is, the original conjunctive wave-length line. Those colors at the same number of increments away from their respective Planckian color plot on a locus approximately paralleling the Planckian locus. With these new coordinates the data given in Tables 4 and 5 for both sides of the Planckian locus were obtained.

TABLE 4.-Illustrating the characteristics of the method for computing correlated color temperature

The colors chosen for these examples are on the spectrum side of the Planckian locus

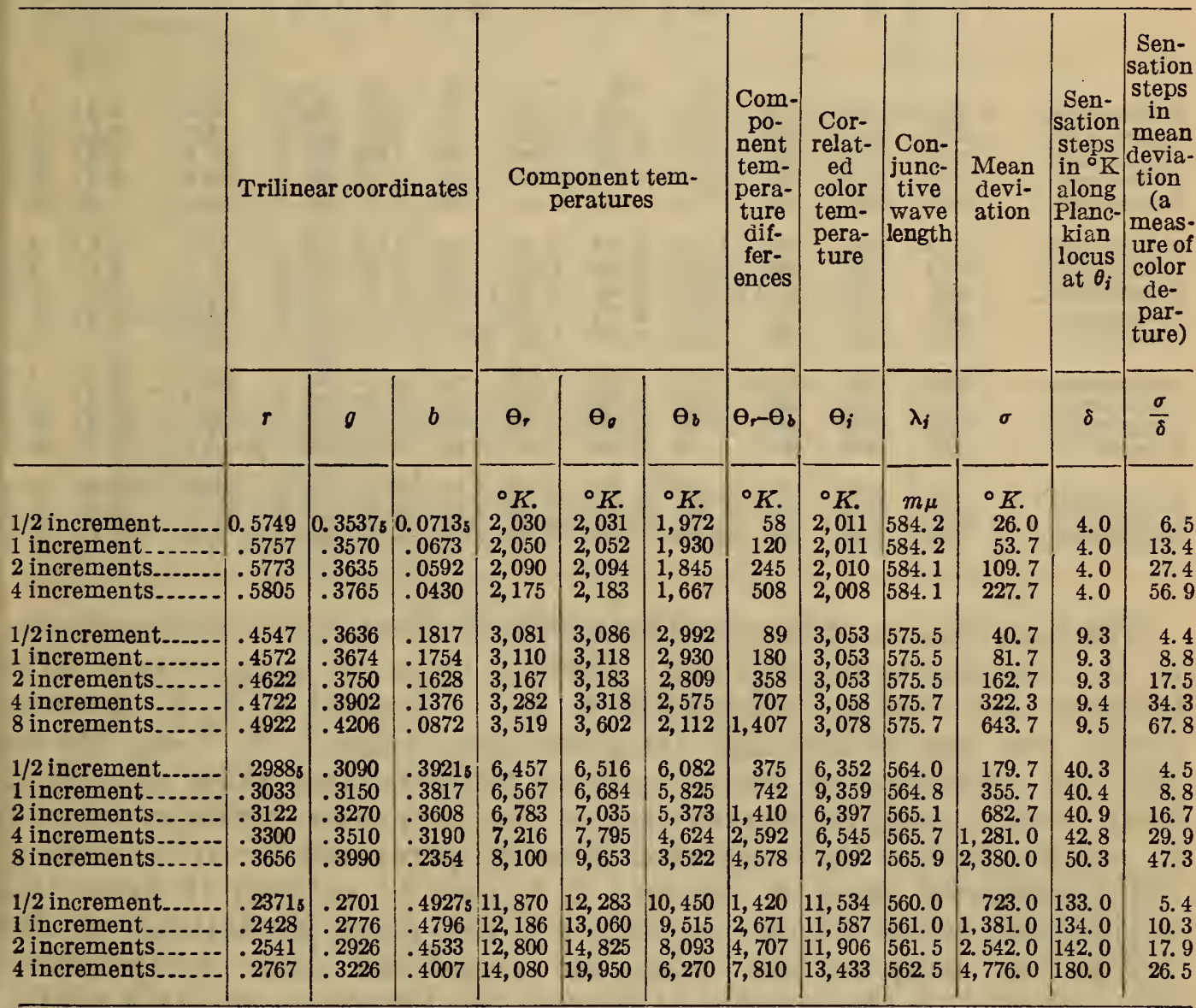


TABLE 5.-Illustrating the characteristics of the method for computing correlated color temperature

The colors chosen for these examples are on the purple side of the Planckian locus

\begin{tabular}{|c|c|c|c|c|c|c|c|c|c|c|c|c|}
\hline & \multicolumn{3}{|c|}{ Trilinear coordinates } & \multicolumn{3}{|c|}{$\begin{array}{l}\text { Component tem- } \\
\text { peratures }\end{array}$} & $\begin{array}{l}\text { Com- } \\
\text { pon- } \\
\text { ent } \\
\text { tem- } \\
\text { pera- } \\
\text { ture } \\
\text { dif- } \\
\text { fer- } \\
\text { ences }\end{array}$ & $\begin{array}{l}\text { Cor- } \\
\text { relat- } \\
\text { ed } \\
\text { color } \\
\text { tem- } \\
\text { pera- } \\
\text { ture }\end{array}$ & $\mid \begin{array}{c}\text { Con- } \\
\text { junc- } \\
\text { tive } \\
\text { wave } \\
\text { length }\end{array}$ & $\begin{array}{l}\text { Mean } \\
\text { devi- } \\
\text { ation }\end{array}$ & $\begin{array}{c}\text { Sen- } \\
\text { sation } \\
\text { steps } \\
\text { in } \mathrm{K} \\
\text { along } \\
\text { Planck- } \\
\text { ian } \\
\text { locus } \\
\text { at } \theta_{i}\end{array}$ & $\begin{array}{l}\text { steps } \\
\text { in } \\
\text { mean } \\
\text { devia } \\
\text { tion(a } \\
\text { meas- } \\
\text { ure of } \\
\text { color } \\
\text { de- } \\
\text { par- } \\
\text { ture) }\end{array}$ \\
\hline & $r$ & $\theta$ & $b$ & $\theta_{p}$ & $\theta_{0}$ & $\theta_{b}$ & $\theta,-\theta_{b}$ & $\boldsymbol{\theta}_{j}$ & $\lambda_{i}$ & $\sigma$ & $\delta$ & $\frac{\sigma}{\delta}$ \\
\hline $\begin{array}{l}4 \text { increment. } \\
8 \text { increment. }\end{array}$ & $\begin{array}{l}0.57325 \\
.5725 \\
.5710 \\
.5680 \\
.5620\end{array}$ & $\left|\begin{array}{c}0.34725 \\
.3440 \\
.3375 \\
.3245 \\
.2985\end{array}\right|$ & $\begin{array}{l}0.0795 \\
.0835 \\
.0915 \\
.1075 \\
.1395\end{array}$ & $\begin{array}{l}{ }^{\circ} K . \\
1,991 \\
1,972 \\
1,933 \\
1,858 \\
1,715\end{array}$ & $\begin{array}{c}{ }^{\circ} \mathrm{K} . \\
1,991 \\
1,970 \\
1,929 \\
1,852 \\
1,704\end{array}$ & $\begin{array}{c}{ }^{\circ} K . \\
2,050 \\
2,090 \\
2,166 \\
2,316 \\
2,615\end{array}$ & \begin{tabular}{r|}
${ }^{\circ} K$ \\
59 \\
118 \\
233 \\
458 \\
900
\end{tabular} & $\begin{array}{c}{ }^{\circ} K . \\
2,011 \\
2,011 \\
2,009 \\
2,009 \\
2,011\end{array}$ & \begin{tabular}{|}
$m_{\mu}$ \\
584.1 \\
584.1 \\
584.3 \\
584.2 \\
584.1
\end{tabular} & $\begin{array}{r}{ }^{\circ} K . \\
26.3 \\
53.0 \\
104.3 \\
205.0 \\
402.3\end{array}$ & $\begin{array}{l}4.0 \\
4.0 \\
4.0 \\
4.0 \\
4.0\end{array}$ & $\begin{array}{r}6 . \\
13 . \\
26 . \\
51 . \\
100 .\end{array}$ \\
\hline $\begin{array}{l}1 / 2 \text { increment. } \\
1 \text { increment. } \\
2 \text { increment.- } \\
4 \text { increment. } \\
8 \text { increment. }\end{array}$ & $\begin{array}{l}.4496 \\
.4472 \\
.4424 \\
.4328 \\
.4136\end{array}$ & $\begin{array}{l}.3560 \\
.3522 \\
.3446 \\
.3294 \\
.2990\end{array}$ & $\begin{array}{l}.1944 \\
.2006 \\
.2130 \\
.2378 \\
.2874\end{array}$ & $\begin{array}{l}3,027 \\
2,998 \\
2,939 \\
2,822 \\
2,594\end{array}$ & $\begin{array}{l}3,024 \\
2,990 \\
2,923 \\
2,794 \\
2,546\end{array}$ & $\begin{array}{l}3,116 \\
3,178 \\
3,310 \\
3,584 \\
4,211\end{array}$ & $\begin{array}{r}89 \\
180 \\
371 \\
762 \\
1,617\end{array}$ & $\begin{array}{l}3,056 \\
3,055 \\
3,057 \\
3,067 \\
3,117\end{array}$ & $\begin{array}{l}575.5 \\
575.4 \\
575.3 \\
574.9 \\
573.8\end{array}$ & $\begin{array}{r}40.3 \\
81.7 \\
168.3 \\
345.0 \\
729.3\end{array}$ & $\begin{array}{l}9.3 \\
9.3 \\
9.3 \\
9.4 \\
9.7\end{array}$ & $\begin{array}{r}8 . \\
18 . \\
36 . \\
75 .\end{array}$ \\
\hline $\begin{array}{l}1 / 2 \text { increment. } \\
1 \text { increment. } \\
2 \text { increment... } \\
4 \text { increment... }\end{array}$ & $\begin{array}{l}.2896_{5} \\
.2855 \\
.2772 \\
.2606\end{array}$ & $\begin{array}{l}.2968 \\
.2910 \\
.2794 \\
.2562\end{array}$ & $\begin{array}{l}.4135_{5} \\
.4235 \\
.4434 \\
.4832\end{array}$ & $\begin{array}{l}6,254 \\
6,134 \\
5,903 \\
5,451\end{array}$ & $\begin{array}{l}6,197 \\
6,033 \\
5,714 \\
5,124\end{array}$ & $\begin{array}{l}6,680 \\
7,000 \\
7,733 \\
9,860\end{array}$ & $\begin{array}{r}426 \\
866 \\
1,830 \\
4,409\end{array}$ & $\begin{array}{l}6,377 \\
6,389 \\
6,450 \\
6,812\end{array}$ & $\begin{array}{l}564.1 \\
563.7 \\
562.5 \\
558.4\end{array}$ & $\begin{array}{r}202.0 \\
407.3 \\
855.3 \\
2,032.0\end{array}$ & $\begin{array}{l}40.7 \\
40.8 \\
41.6 \\
46.4\end{array}$ & $\begin{array}{r}5 . \\
10 . \\
20 . \\
43 .\end{array}$ \\
\hline $\begin{array}{l}1 / 2 \text { increment } \\
1 \text { increment.-. } \\
2 \text { increment... }\end{array}$ & $\begin{array}{l}.2241_{5} \\
.2202 \\
.2123\end{array}$ & $\begin{array}{l}.2540_{5} \\
.2479 \\
.2356\end{array}$ & $\begin{array}{l}.5218 \\
.5319 \\
.5521\end{array}$ & $\left|\begin{array}{l}11,620 \\
11,080 \\
10,090\end{array}\right|$ & $\begin{array}{r}11,260 \\
10,450 \\
9,080\end{array}$ & $\begin{array}{l}13,483 \\
15,100 \\
20,260\end{array}$ & $\begin{array}{l}1,863 \\
4,020 \\
10,170\end{array}$ & $\begin{array}{l}12,121 \\
12,210 \\
13,143\end{array}$ & $\begin{array}{l}557.0 \\
555.3 \\
548.2\end{array}$ & $\begin{array}{r}908.0 \\
1,927.0 \\
4,744.0\end{array}$ & $\begin{array}{l}147.0 \\
149.0 \\
173.0\end{array}$ & $\begin{array}{l}6 . \\
12 . \\
27 .\end{array}$ \\
\hline
\end{tabular}

The first column of these tables serves to identify the points chosen by the increments as described; the next three columns contain their trilinear coordinates; columns 5,6 , and 7 headed $\theta_{r}, \theta_{g}, \theta_{b}$, respectively, are the component temperatures; column $8, \theta_{r}-\theta_{b}$, shows that the separation of the component temperatures along the black body locus is roughly proportional to the increments given in column 1 ; column 9 gives the correlated color temperatures for colors having the coordinates given in columns 2,3 , and 4 ; column 10 gives the conjunctive wave length, column 11, the mean deviations of the component temperatures from the correlated color temperature $\left(\theta_{j}\right)$; column 12, the number of degrees the Planckian radiator would have to be increased or decreased in temperature to give a difference in color which would be just perceptible, as given by the expression $\delta=K \theta^{2}$; and column 13 , the mean deviation divided by $\delta$. Note that these last values also increase roughly by powers of 2 .

These tables show that the spread of the component temperatures along the Planckian locus, the mean deviation, and $\frac{\sigma}{\delta}$ are approximately proportional to the difference between the trilinear coordinates of the Planckian and the non-Planckian colors. In Table 4 the $\lambda$, values increase slightly with the number of "increments" indicating that the isotemperature line is slightly curved. Furthermore, the curvature becomes more pronounced as the temperature 
increases, and $\lambda_{j}$ changes with $\theta_{j}$. In Table 5 , referring to colors on the purple side of the Planckian locus, the conjunctive wave length decreases as the distance from the Planckian locus increases; indicating that the isotemperature line continues to curve in the same direction as on the spectrum side of the Planckian locus.

\section{CONJUNCTIVE WAVE LENGTH AS A FUNCTION OF CORRELATED COLOR TEMPERATURE}

If the conjunctive wave lengths corresponding to a series of given Planckian colors be marked on the spectrum locus, (on a $r-b$ diagram) lines joining these points with the corresponding Planckian points

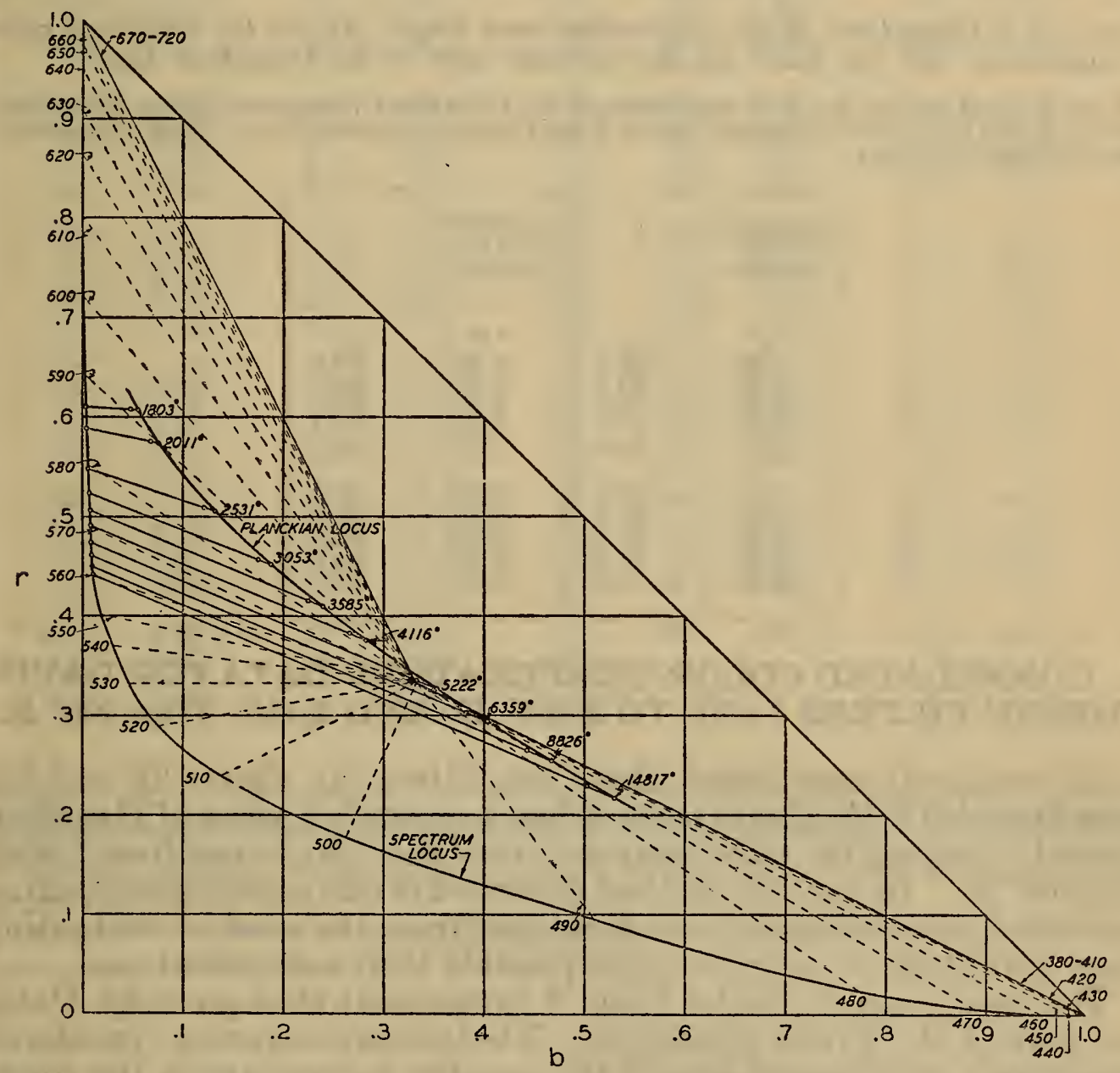

FIGURE 3.-Illustrating the variation of conjunctive wave length $\left(\lambda_{i}\right)$ with correlated color temperature using the data in Table 6 and plotted on a $r-b$ diagram with mean sun as the "neutral" stimulus

will indicate roughly the direction of color departure. A diagram of this type (shown in fig. 3) is of practical utility because, since these lines on the plot are nearly parallel, one may obtain, graphically, an approximate value of the correlated color temperature of any color lying in the neighborhood of one of these lines and near the Planckian locus.

To obtain this relation a series of non-Planckian colors was selected (by the method previously described for Table 5) the locus of which parallels the Planckian locus at a distance of "one increment." 
From the equation of the line joining the points, representing respectively the Planckian and the non-Planckian colors, the intercept on the $r$ axis is found. Drawing a line from this point through the two given points, its intersection with the spectrum locus is found graphically. From the coordinates of this intersection the corresponding spectrum wave length (conjunctive wave length) is found by way of a table (not published) containing values of $\frac{g-b}{r-b}, \frac{g-r}{b-r}$ or $\frac{r-g}{b-g}$ for each millimicron of wave length. In this way the values given in Table 6 were found. The material of Table 6 is applied in figure 3 , an $r-b$ projection of the trilinear diagram.

TABLE 6.-Dependence of the conjunctive wave length $\left(\boldsymbol{\lambda}_{i}\right)$ on the correlated color temperature $\left(\theta_{j}\right)$ for colors on the spectrum side of the Planckian locus

It will be noted that tha hue of the color corresponding to the added homogensous radiant energy does not vary greatly (green yellow to orange); that is, $\lambda_{i}$ has a range of only about $30 \mathrm{~m} \mu$. These values are further illustrated in Figure 3.

\begin{tabular}{|c|c||c|c|}
\hline $\begin{array}{c}\theta \text { correlated } \\
\text { color tem- } \\
\text { perature }\end{array}$ & $\lambda_{i}$ & $\begin{array}{c}\theta \text { correlated } \\
\text { color tem- } \\
\text { perature }\end{array}$ & $\lambda_{j}$ \\
\hline & & ${ }^{\circ} K$ & \\
\hline${ }^{\circ} K$ & $m_{\mu}$ & ${ }^{\prime}$ & \\
1,803 & 586.5 & 6,945 & 564.0 \\
2,011 & 584.2 & 7,549 & 563.2 \\
2,532 & 579.5 & 8,181 & 562.6 \\
3,053 & 575.5 & 8,826 & 562.1 \\
3,585 & 572.9 & 9,483 & 561.4 \\
& & & \\
4,116 & 570.2 & 10,187 & 561.0 \\
4,663 & 568.8 & 10,876 & 561.2 \\
5,222 & 567.0 & 11,587 & 561.0 \\
5,781 & 565.8 & 13,161 & 560.3 \\
6,359 & 564.8 & 14,817 & 560.3 \\
\hline
\end{tabular}

\section{CORRELATED COLOR TEMPERATURE DATA FOR DAVIS- GIBSON FILTERS $2,450^{\circ}$ TO $3,500^{\circ} \mathrm{K}$. AND $2,450^{\circ}$ TO $6,500^{\circ} \mathrm{K}$.}

As previously mentioned, these two filters (4; charts 37 and 38 ) were intended to duplicate more or less accurately a series of Planckian colors by varying the color temperature of the light source from $2,000^{\circ}$ to $3,100^{\circ} \mathrm{K}$. In fact, the method described in this paperfor computing correlated color temperatures developed from the need of calibrating these two filters in order to make possible their convenient use.

The data given in Tables 7 and 8 supplement that given by Davis and Gibson (4; Tables 15 and 16). The last three columns, considered collectively, give a good idea of the possible uncertainty in the correlated color temperatures given in the second column. 
TABLE 7.-Correlated color temperature data as functions of the light-source temperature of the Davis-Gibson filter (chart No. 37 and Table 14 of B. S. Misc. Pub. No. 114) designed to convert $2,450^{\circ}$ to $3,500^{\circ} \mathrm{K}$

\begin{tabular}{|c|c|c|c|c|c|c|c|c|c|}
\hline $\begin{array}{l}\text { Color } \\
\text { temper- } \\
\text { ature of } \\
\text { light } \\
\text { source }\end{array}$ & $\begin{array}{l}\text { Correlat- } \\
\text { ed } 1 \text { color } \\
\text { tempera- } \\
\text { ture of } \\
\text { source } \\
\text { and filter } \\
\text { combi- } \\
\text { nation }\end{array}$ & $\begin{array}{c}\sigma \text { (mean }^{2} \\
\text { devia- } \\
\text { tion) }\end{array}$ & $\delta\left(K \theta^{2}\right)$ & $\begin{array}{c}\frac{\sigma}{\delta} \\
\text { sensation } \\
\text { steps }^{3}\end{array}$ & $\begin{array}{l}\text { Color } \\
\text { temper- } \\
\text { ature of } \\
\text { light } \\
\text { source }\end{array}$ & $\begin{array}{l}\text { Correlat- } \\
\text { ed } 1 \text { color } \\
\text { tempera- } \\
\text { ture of } \\
\text { source } \\
\text { and filter } \\
\text { combi- } \\
\text { nation }\end{array}$ & 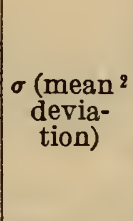 & $\delta\left(K \theta^{2}\right)$ & $\begin{array}{c}{ }_{\bar{\delta}}^{\sigma} \\
\text { sensation } \\
\text { steps }^{3}\end{array}$ \\
\hline $\begin{array}{r}\circ K \\
2,000 \\
20 \\
40 \\
60 \\
80\end{array}$ & $\begin{array}{c}{ }^{\circ} K \\
2,649 \\
2,685 \\
2,721 \\
2,757 \\
2,793\end{array}$ & $\begin{array}{r}\circ K \\
8 \\
7 \\
7 \\
6 \\
5\end{array}$ & $\begin{array}{l}7.0 \\
7.2 \\
7.4 \\
7.6 \\
7.8\end{array}$ & $\begin{array}{l}1.1 \\
1.0 \\
.9 \\
.8 \\
.7\end{array}$ & $\begin{array}{r}{ }^{\circ} K \\
2,600 \\
20 \\
40 \\
60 \\
80\end{array}$ & $\begin{array}{l}{ }^{\circ} K \\
3,817 \\
3,859 \\
3,903 \\
3,947 \\
3,992\end{array}$ & $\begin{array}{r}\circ K \\
7 \\
8 \\
9 \\
9 \\
10\end{array}$ & $\begin{array}{l}14.6 \\
14.9 \\
15.2 \\
15.6 \\
15.9\end{array}$ & $\begin{array}{l}.5 \\
.5 \\
.6 \\
.6 \\
.6\end{array}$ \\
\hline $\begin{array}{r}2,100 \\
20 \\
40 \\
60 \\
80\end{array}$ & $\begin{array}{l}2,829 \\
2,865 \\
2,901 \\
2,939 \\
2,976\end{array}$ & $\begin{array}{l}5 \\
4 \\
4 \\
3 \\
3\end{array}$ & $\begin{array}{l}8.0 \\
8.2 \\
8.4 \\
8.6 \\
8.9\end{array}$ & $\begin{array}{l}.7 \\
.6 \\
.5 \\
.5 \\
.4\end{array}$ & $\begin{array}{r}2,700 \\
20 \\
40 \\
60 \\
80\end{array}$ & $\begin{array}{l}4,038 \\
4,082 \\
4,126 \\
4,171 \\
4,216\end{array}$ & $\begin{array}{l}10 \\
11 \\
11 \\
12 \\
13\end{array}$ & $\begin{array}{r}16.3 \\
16.7 \\
17.0 \\
17.4 \\
17.8\end{array}$ & $\begin{array}{l}.6 \\
.6 \\
.7 \\
.7\end{array}$ \\
\hline $\begin{array}{r}2,200 \\
20 \\
40 \\
60 \\
80\end{array}$ & $\begin{array}{l}3,012 \\
3,051 \\
3,088 \\
3,127 \\
3,165\end{array}$ & $\begin{array}{l}3 \\
3 \\
3 \\
2 \\
2\end{array}$ & $\begin{array}{r}9.1 \\
9.3 \\
9.5 \\
9.8 \\
10.0\end{array}$ & $\begin{array}{l}.4 \\
.4 \\
.3 \\
.3 \\
.3\end{array}$ & $\begin{array}{r}2,800 \\
20 \\
40 \\
60 \\
80\end{array}$ & $\begin{array}{l}4,262 \\
4,309 \\
4,356 \\
4,404 \\
4,453\end{array}$ & $\begin{array}{l}14 \\
14 \\
15 \\
16 \\
17\end{array}$ & $\begin{array}{l}18.2 \\
18.6 \\
19.0 \\
19.4 \\
19.8\end{array}$ & $\begin{array}{l}.7 \\
.7 \\
.8 \\
.8 \\
.9\end{array}$ \\
\hline $\begin{array}{r}2,300 \\
20 \\
40 \\
60 \\
80\end{array}$ & $\begin{array}{l}3,202 \\
3,242 \\
3,281 \\
3,320 \\
3,359\end{array}$ & $\begin{array}{l}2 \\
2 \\
1 \\
1 \\
1\end{array}$ & $\begin{array}{l}10.3 \\
10.5 \\
10.8 \\
11.0 \\
11.3\end{array}$ & $\begin{array}{l}.2 \\
.2 \\
.2 \\
.1 \\
.1\end{array}$ & $\begin{array}{r}2,900 \\
20 \\
40 \\
60 \\
80\end{array}$ & $\begin{array}{l}4,502 \\
4,551 \\
4,600 \\
4,650 \\
4,700\end{array}$ & $\begin{array}{l}18 \\
18 \\
19 \\
20 \\
21\end{array}$ & $\begin{array}{l}20.3 \\
20.7 \\
21.2 \\
21.6 \\
22.1\end{array}$ & $\begin{array}{r}.9 \\
.9 \\
.9 \\
1.0 \\
1.0\end{array}$ \\
\hline $\begin{array}{r}2,400 \\
20 \\
40 \\
60 \\
80\end{array}$ & $\begin{array}{l}3,398 \\
3,439 \\
3,480 \\
3,521 \\
3,563\end{array}$ & $\begin{array}{l}1 \\
0 \\
0 \\
0 \\
1\end{array}$ & $\begin{array}{l}11.5 \\
11.8 \\
12.1 \\
12.4 \\
12.7\end{array}$ & $\begin{array}{l}.1 \\
.1 \\
.0 \\
.0 \\
.1\end{array}$ & $\begin{array}{r}3,000 \\
20 \\
40 \\
60 \\
80\end{array}$ & $\begin{array}{l}4,751 \\
4,803 \\
4,855 \\
4,908 \\
4,961\end{array}$ & $\begin{array}{l}22 \\
23 \\
25 \\
28 \\
31\end{array}$ & $\begin{array}{l}22.6 \\
23.1 \\
23.6 \\
24.1 \\
24.6\end{array}$ & $\begin{array}{l}1.0 \\
1.0 \\
1.0 \\
1.1 \\
1.2\end{array}$ \\
\hline $\begin{array}{r}2,500 \\
20 \\
40 \\
60 \\
80\end{array}$ & $\begin{array}{l}3,605 \\
3,647 \\
3.688 \\
3,730 \\
3,773\end{array}$ & $\begin{array}{l}2 \\
3 \\
4 \\
5 \\
6\end{array}$ & $\begin{array}{l}13.0 \\
13.3 \\
13.6 \\
13.9 \\
14.2\end{array}$ & $\begin{array}{l}.1 \\
.2 \\
.3 \\
.4 \\
.5\end{array}$ & 3,100 & 5,015 & 34 & 25.2 & 1.3 \\
\hline
\end{tabular}

1 The values in the second and third columns were obtained from large-scale graphs plotted from data computed for every $100^{\circ}$ color temperature of the light source. It is impossible at this time to state the accuracy of the color temperatures given in the second column. They are given to the nearest degree to facilitate interpolation.

The mean deviations $(\sigma)$ plot somewhat irregularly and their accuracy is not, in general, considered to be better than $\pm 1^{\circ}$.

${ }^{3}$ To preserve continuity of the values $\frac{\sigma}{\delta}$, the mean deviations $(\sigma)$ used were smoothed to an extent beyond those given in the third column.

$$
74280-31-5
$$


TABLE 8.-Correlated color temperature data as functions of the light source temperature of the Davis-Gibson filter (chart No. 38 and Table 15 of B. S. Misc. Pub. No. 114) designed to convert $2,450^{\circ}$ to $6,500^{\circ} \mathrm{K}$.

\begin{tabular}{|c|c|c|c|c|c|c|c|c|c|}
\hline $\begin{array}{l}\text { Color } \\
\text { temper- } \\
\text { ature of } \\
\text { light } \\
\text { source }\end{array}$ & $\begin{array}{l}\text { Correlat- } \\
\text { ed I color } \\
\text { tempera- } \\
\text { ture of } \\
\text { source } \\
\text { and filter } \\
\text { combi- } \\
\text { nation }\end{array}$ & $\begin{array}{c}\sigma \text { (mean }{ }^{2} \\
\text { devia- } \\
\text { tion) }\end{array}$ & $\delta\left(K \theta^{2}\right)$ & $\begin{array}{c}\frac{\sigma}{\delta} \\
\text { sensation } \\
\text { step }\end{array}$ & $\begin{array}{l}\text { Color } \\
\text { temper- } \\
\text { ature of } \\
\text { light } \\
\text { source }\end{array}$ & $\begin{array}{l}\text { Correlat- } \\
\text { ed l color } \\
\text { tempera- } \\
\text { ture of } \\
\text { source } \\
\text { and filter } \\
\text { combi- } \\
\text { nation }\end{array}$ & $\begin{array}{c}\sigma \text { (mean } \\
\text { devia- } \\
\text { tion) }\end{array}$ & $\delta\left(K \theta^{2}\right)$ & $\begin{array}{c}\frac{\sigma}{\delta} \\
\text { sensation } \\
\text { step }\end{array}$ \\
\hline $\begin{array}{r}\circ K . \\
2,000 \\
20 \\
40 \\
60 \\
80\end{array}$ & $\begin{array}{l}{ }^{\circ} K . \\
4,031 \\
4,116 \\
4,201 \\
4,289 \\
4,378\end{array}$ & $\begin{array}{r}\circ K . \\
47 \\
47 \\
47 \\
47 \\
46\end{array}$ & $\begin{array}{l}16 \\
17 \\
18 \\
18 \\
19\end{array}$ & $\begin{array}{l}2.9 \\
2.8 \\
2.6 \\
2.6 \\
2.4\end{array}$ & $\begin{array}{r}\circ K . \\
2,600 \\
20 \\
40 \\
60 \\
80\end{array}$ & $\begin{array}{c}\circ K . \\
7,773 \\
7,976 \\
8,185 \\
8,404 \\
8,632\end{array}$ & $\begin{array}{r}\circ K . \\
66 \\
78 \\
91 \\
105 \\
121\end{array}$ & $\begin{array}{l}60 \\
64 \\
67 \\
71 \\
75\end{array}$ & $\begin{array}{l}1.1 \\
1.2 \\
1.4 \\
1.5 \\
1.6\end{array}$ \\
\hline $\begin{array}{r}2,100 \\
20 \\
40 \\
60 \\
80\end{array}$ & $\begin{array}{l}4,468 \\
4,560 \\
4,656 \\
4,752 \\
4,853\end{array}$ & $\begin{array}{l}45 \\
45 \\
44 \\
42 \\
41\end{array}$ & $\begin{array}{l}20 \\
21 \\
22 \\
23 \\
24\end{array}$ & $\begin{array}{l}2.2 \\
2.1 \\
2.0 \\
1.8 \\
1.7\end{array}$ & $\begin{array}{r}2,700 \\
20 \\
40 \\
60 \\
80\end{array}$ & $\begin{array}{l}8,869 \\
9,123 \\
9,387 \\
9,665 \\
9,957\end{array}$ & $\begin{array}{l}139 \\
160 \\
183 \\
211 \\
244\end{array}$ & $\begin{array}{l}79 \\
83 \\
88 \\
93 \\
99\end{array}$ & $\begin{array}{l}1.8 \\
1.9 \\
2.1 \\
2.3 \\
2.5\end{array}$ \\
\hline $\begin{array}{r}2,200 \\
20 \\
40 \\
60 \\
80\end{array}$ & $\begin{array}{l}4,956 \\
5,060 \\
5,168 \\
5,279 \\
5,393\end{array}$ & $\begin{array}{l}39 \\
37 \\
35 \\
32 \\
28\end{array}$ & $\begin{array}{l}25 \\
26 \\
27 \\
28 \\
29\end{array}$ & $\begin{array}{l}1.6 \\
1.4 \\
1.3 \\
1.1 \\
1.0\end{array}$ & $\begin{array}{r}2,800 \\
20 \\
40 \\
60 \\
80\end{array}$ & $\begin{array}{l}10,252 \\
10,600 \\
10,956 \\
11,320 \\
11,696\end{array}$ & $\begin{array}{l}278 \\
318 \\
360 \\
404 \\
450\end{array}$ & $\begin{array}{l}105 \\
112 \\
120 \\
128 \\
137\end{array}$ & $\begin{array}{l}2.6 \\
2.8 \\
3.0 \\
\text { 3. } 2 \\
\text { 3. } 3\end{array}$ \\
\hline $\begin{array}{r}2,300 \\
20 \\
40 \\
60 \\
80\end{array}$ & $\begin{array}{l}5,511 \\
5,632 \\
5,757 \\
5,884 \\
6,014\end{array}$ & $\begin{array}{l}25 \\
23 \\
21 \\
18 \\
16\end{array}$ & $\begin{array}{l}30 \\
32 \\
33 \\
35 \\
36\end{array}$ & $\begin{array}{l}.8 \\
.7 \\
.6 \\
.5 \\
.4\end{array}$ & $\begin{array}{r}2,900 \\
20 \\
40 \\
60 \\
80\end{array}$ & $\begin{array}{l}12,090 \\
12,517 \\
12,979 \\
13,482 \\
14,030\end{array}$ & $\begin{array}{l}500 \\
559 \\
624 \\
700 \\
789\end{array}$ & $\begin{array}{l}146 \\
157 \\
168 \\
182 \\
197\end{array}$ & $\begin{array}{l}3.4 \\
3.6 \\
3.7 \\
3.8 \\
4.0\end{array}$ \\
\hline $\begin{array}{r}2,400 \\
20 \\
40 \\
60 \\
80\end{array}$ & $\begin{array}{l}6,143 \\
6,286 \\
6,430 \\
6,578 \\
6,728\end{array}$ & $\begin{array}{r}13 \\
8 \\
2 \\
4 \\
11\end{array}$ & $\begin{array}{l}38 \\
40 \\
41 \\
43 \\
45\end{array}$ & $\begin{array}{l}.3 \\
.2 \\
.0 \\
.1 \\
.2\end{array}$ & $\begin{array}{r}3,000 \\
20 \\
40 \\
60 \\
80\end{array}$ & $\begin{array}{l}14,631 \\
15,299 \\
16,032 \\
16,844 \\
17,737\end{array}$ & $\begin{array}{r}892 \\
1,020 \\
1,169 \\
1,346 \\
1,557\end{array}$ & $\begin{array}{l}214 \\
234 \\
257 \\
284 \\
315\end{array}$ & $\begin{array}{l}4.2 \\
4.4 \\
4.5 \\
4.7 \\
4.9\end{array}$ \\
\hline $\begin{array}{r}2,500 \\
20 \\
40 \\
60 \\
80\end{array}$ & $\begin{array}{l}6,884 \\
7,047 \\
7,217 \\
7,394 \\
7,580\end{array}$ & $\begin{array}{l}16 \\
23 \\
32 \\
43 \\
54\end{array}$ & $\begin{array}{l}47 \\
50 \\
52 \\
55 \\
57\end{array}$ & $\begin{array}{l}.3 \\
.5 \\
.6 \\
.8 \\
.9\end{array}$ & 3,100 & 18,667 & 1,778 & 348 & 5.1 \\
\hline
\end{tabular}

1 The values in the second and third columns were obtained from large-scale graphs plotted from data computed for every $100^{\circ}$ color temperature of the light source. It is impossible at this time to state the accuracy of the color temperatures given in the second column. They are given to the nearest degree to facilitate interpolation.

2 The mean deviations $(\sigma)$ plot somewhat irregularly and their accuracy is not, in general, considered to be better than $\pm 1^{\circ}$.

\section{BIBLIOGRAPHY}

1. Hyde, Edward P., A New Determination of the Selective Radiation from Tantalum (abstract), Phys. Rev., vol. 32 p. $632 ; 1911$.

2. Priest, I. G., The Colorimetry and Photometry of Daylight and Incandescent Illuminants by the Method of Rotatory Dispersion, J. Opt. Soc. Am. and Rev. Sci. Inst., vol. 7 No. 12, pp. 1175-1209; 1923.

3. Forsythe, W. E., Color Match and Spectral Distribution, J. Opt. Soc. Am. and Rev. Sci. Inst., vol. 7 No. 12 , pp. 1115-1121; 1923.

4. Davis, Raymond, and Gibson, K. S., Filters for the Reproduction of Sunlight and Daylight and the Determination of Color Temperature. B. S. Mis. Publication No. 114 (45 cents). ${ }^{7}$

5. Troland, L. T. report of O. S. A. Committee on Colorimetry for 1920-21, J. Opt. Soc. Am. and Rev. Sci. Inst., vol. 6 No. 6, pp. 527-596; 1922.

6. Gibson, K. S., report of O. S. A. Committee on Spectrophotometry for 1922-23, J. Opt. Soc. Am. and Rev. Sci. Inst., vol. 10, pp. 169-241; 1925.

7 National Burcau of Standards official publications may be ohtained from the Superintendent of Documents, Government Printing Ofice, Washington, D. C., postpaid at the prices given. 
7. Proceedings, International Commission on Illumination, Sixth Mecting, Geneva, 1924, pp. 67 and 232-238; Cambridge University Press, 1926, see also Gibson, K. S. and Tyndall, E. P. T., The Visibility of Radiant Energy, B. S. Sci. Paper No. 475 (15 cents). ${ }^{7}$

8. Judd, Deane B., Chromatic Visibility Coefficients by the Method of Least Squares, J. Opt. Soc. Am. and Rev. Sci. Inst., vol. 10 No.6, pp.635-651; 1925.

9. Judd, D. B., Precision of Color Temperature Measurements under Various Observing Conditions; A New Color Comparator for Incandescent Lamps, B. S. Jour. Research; vol. 5, p. 1175; 1930.7

10. Priest, I. G., and Brickwedde, F. G., The Minimum Perceptible Colorimetric Purity as a Function of Dominant Wave Length with Sunlight as Neutral Standard, J. Opt. Soc. Am. and Rev. Sci. Inst., vol. 13, p. 306; 1926.

11. Priest, I. G. Measurements of the Cclor Temperature of the More Efficient Artificial Light Sources by the Method of Rotatory Dispersion, B. S. Sci. Paper No. 443; 1922. 7

12. Priest, I. G., Standard Artificial Sunlight for Colorimetric Purposes, J. Opt. Soc. Am., and Rev. Sci. Inst., vol. 12 No. 5, p. 480; 1926.

Washington, November 1, 1930.

7 National Bureau of Standards official publications may be obtained from the Superintendent of Documents, Government Printing Office, Washington, D. C., postpaid at the prices given. 